\title{
Protostellar collapse: a comparison between smoothed particle hydrodynamics and adaptative mesh refinement calculations
}

\author{
B. Commerçon ${ }^{1,2,3}$, P. Hennebelle ${ }^{3}$, E. Audit ${ }^{2}$, G. Chabrier ${ }^{1}$, and R. Teyssier ${ }^{2}$ \\ 1 École Normale Supérieure de Lyon, Centre de recherche Astrophysique de Lyon (UMR 5574 CNRS), 46 allée d'Italie, \\ 69364 Lyon Cedex 07, France \\ e-mail: benoit. commercon@ens-lyon. fr \\ 2 Laboratoire AIM, CEA/DSM - CNRS - Université Paris Diderot, IRFU/SAp, 91191 Gif sur Yvette, France \\ ${ }^{3}$ Laboratoire de radioastronomie millimétrique (UMR 8112 CNRS), École Normale Supérieure et Observatoire de Paris, \\ 24 rue Lhomond, 75231 Paris Cedex 05, France
}

Received 31 August 2007 / Accepted 16 January 2008

ABSTRACT

\begin{abstract}
Context. The rapid development of parallel supercomputers is enabling the detailed study of the collapse and the fragmentation of prestellar cores with increasingly accurate numerical simulations. Due to the advances also in sub-millimeter observation technology, we are now able to consider many different modes of low-mass star formation using observations of a range of initials conditions. The challenge for the simulations is to reproduce the observational results.

Aims. Two main numerical methods, namely AMR and SPH, are widely used to simulate the collapse and the fragmentation of prestellar cores. We thoroughly compare these two methods within their standard framework.

Methods. We use the AMR code RAMSES and the SPH code DRAGON. Our simplified physical model consists of an isothermal sphere rotating about the $z$-axis. First we study the conservation of angular momentum as a function of the resolution. Then, we explore a wide range of simulation parameters to study the fragmentation of prestellar cores.

Results. There appears to be convergence between the two methods, provided numerical resolution in each case is sufficient. We deduced numerical resolution criteria adapted to our physical cases, in terms of resolution per Jeans mass, for an accurate description of the formation of protostellar cores. This convergence is encouraging for future work in simulations of low-mass star formation, providing the aforementioned criteria are fulfilled.
\end{abstract}

Key words. stars: formation - methods: numerical - hydrodynamics

\section{Introduction}

The study of star formation considers an incredibly wide range of mass and length scales. Although it is established that stars form in dense cores, their non-linear evolution makes it difficult to perform accurate calculations of the collapse and the fragmentation of a prestellar core. The star formation process is the outcome of complex gas dynamics involving non-linear interactions of gravity, turbulence, magnetic field and radiation. The early, pioneering theoretical works of Larson (1969), Penston (1969) or Shu (1977) demonstrated the high complexity of gravitational collapse. Klein et al. (2007) recently stated that developing a theory for low-mass star formation remains one of the most elusive and important goals of theoretical astrophysics. The computational challenge is to consider effectively the extensive ranges of spatial and density scale. Following the gravitational collapse while resolving precisely the Jeans length, which scales as $\lambda_{\mathrm{J}} \propto \rho^{-1 / 2}$ for an isothermal gas, is a major difficulty for numerical simulations.

Different numerical-simulation, techniques are used to study star formation, which have included increasingly more complex physics. One difficulty is always the validation of the numerical methods used to study low-mass star formation. There are two distinct numerical methods that provide sufficient accuracy:

1. AMR: Adaptive Mesh Refinement method for Eulerian grids.
2. SPH: Smoothed Particle Hydrodynamics method for a Lagrangian approach.

No systematic comparison between the two methods has yet been completed for low-mass star formation calculations. Many numerical studies have compiled common test calculations for convergence testing, and intercode comparison. The most famous model was first calculated by Boss \& Bodenheimer (1979) and since then, it has been recalculated by several authors with even higher spatial resolution (e.g. Bate \& Burkert 1997; Truelove et al. 1998; Kitsionas \& Whitworth 2002; Arreaga-García et al. 2007). The SPH approach has generated a lot of detailed investigations on the influence of the number of particles and neighbors (Lombardi et al. 1999; Rasio 1999; Attwood et al. 2007), and criteria for numerical convergence have been extracted from these studies. Nelson (2006) performed a large investigation of the influence of these parameters on disk fragmentation, and concluded that the better the resolution the later the fragmentation. Dehnen (2001) investigated the optimal gravitational force softening necessary in three-dimensional $N$-body codes. Bate \& Burkert (1997) provide a minimum-resolution, criterion for SPH calculations with self-gravity to accurately model fragmentation. Fewer studies have been completed for AMR because AMR codes have become available only recently. Truelove et al. (1997) provide an empirical criterion for the Jeans-length resolution in AMR calculations to avoid spurious numerical fragmentation. 
There have not been many direct comparison between SPH and AMR calculations. Comparison in the context of cosmological simulations has been completed by the Santa Barbara Cluster Comparison Project (Frenk et al. 1999). Using the AMR method, Fromang et al. (2006) compares quite successfully hydrodynamical collapse calculations with SPH results of Hosking \& Whitworth (2004).

In the present paper, we thoroughly compare the two approaches in the context of low-mass prestellar core formation. The main goal of this paper is to investigate whether convergence can be achieved between the two methods. We have conducted calculations over a wide range of numerical resolution parameters, in order to study the dependency of angular momentum conservation and fragmentation on physical and numerical initial conditions. We then determine the resolution criteria required to accurately describe prestellar core formation.

The paper is organized as follows: in Sect. 2 we briefly introduce our collapse model. In Sect. 3, we present the two codes used in our comparative study, our initial numerical conditions, and the criteria we fulfill to resolve gravitational collapse. The problem of angular momentum conservation is examined in detail in Sect. 4. In Sect. 5, we tackle the fragmentation issue and explore the dependency of the results on the numerical parameters. First, we study the numerical convergence of AMR and SPH calculations separately. Then, we compare the converged calculations for each code. This convergence study is completed for different test cases. In Sect. 6, we present our conclusions. For each method, we discuss the numerical criteria required to provide an accurate description of both gravitational collapse and fragmentation.

The convention in this paper is to call "particles" the SPH particles and "cells" the AMR cells in order to avoid confusion.

\section{Definitions of the test cases}

\subsection{Model}

To make comparison between codes easier, we adopt simple initial conditions, similar to those chosen in previous studies (e.g. Boss \& Bodenheimer 1979; Bate \& Burkert 1997). We consider a uniform-density sphere of molecular gas of initial radius $R_{0}$, rotating about the $z$-axis with a uniform angular velocity $\Omega_{0}$, in order to minimize the loss of angular momentum due to friction. We fix the cloud mass at $M_{0}=1 M_{\odot}$ and the temperature at $10 \mathrm{~K}$. For a mixture of molecular hydrogen, helium and heavy elements, this corresponds to an isothermal sound speed of $C_{0} \sim 0.19 \mathrm{~km} \mathrm{~s}^{-1}$. For the case where fragmentation occurs, we use an $m=2$ azimuthal density perturbation.

The initial energy balance of our model is determined by two dimensionless parameters corresponding to the ratio between the thermal energy and the gravitational energy

$\alpha=\frac{5}{2} \frac{R_{0} k T}{G M_{0} \mu m_{\mathrm{H}}}$,

and to the ratio of the rotational and the gravitational energy

$\beta=\frac{1}{3} \frac{R_{0}^{3} \Omega_{0}^{2}}{G M_{0}}$.

Since we use a constant initial mass of $1 M_{\odot}$ and a constant temperature, providing the value of the parameter $\alpha$, gives the sphere radius $R_{0}$. The higher $\alpha$ becomes, the larger $R_{0}$ is. The angular velocity is given by the parameter $\beta$.

\subsection{The barotropic equation of state}

In order to mimic the thermal behaviour of a star-forming gas, we use a barotropic equation of state (cf. Bonnell 1994). Tohline (1982) and Masunaga \& Inutsuka (2000) showed that the core closely follows a barotropic equation of state, providing a good approximation without resolving radiative transfer. We use

$$
\frac{P}{\rho}=C_{0}^{2}\left[1+\left(\frac{\rho}{\rho_{\mathrm{c}}}\right)^{2 / 3}\right]
$$

where $C_{\mathrm{s}}$ is the sound speed and $\rho_{\mathrm{c}}=10^{-13} \mathrm{~g} \mathrm{~cm}^{-3}$ is the critical density which corresponds to the transition from an isothermal to an adiabatic state (Larson 1969).

At low densities, $\rho \ll \rho_{\mathrm{c}}, C_{\mathrm{s}} \sim C_{0}=0.19 \mathrm{~km} \mathrm{~s}^{-1}$. The molecular gas is able to radiate freely by thermally coupling to the dust and remains isothermal at $10 \mathrm{~K}$. At high densities $\rho>\rho_{\mathrm{c}}$, we assume that the cooling due to radiative transfer is trapped by the dust opacity. Therefore, $P \propto \rho^{5 / 3}$ which corresponds to an adiabatic, monoatomic gas with adiabatic exponent $\gamma=5 / 3$. Molecular hydrogen behaves like a monoatomic gas until the temperature reaches several hundred Kelvin. This is because the rotational degrees of freedom are not excited at lower temperatures, and hence $\gamma=5 / 3$ is the appropriate adiabatic exponent (Whitworth \& Clarke 1997; Masunaga \& Inutsuka 2000).

\section{Numerical methods and initial conditions}

\subsection{AMR: Adaptive Mesh Refinement}

\subsubsection{A brief history}

The Adaptive Mesh Refinement method is one of the most promising numerical methods to solve the fluid equations. The technique was first introduced in Berger \& Oliger (1984). Originally, the AMR method was an Eulerian hydrodynamical scheme, with a hierarchy of nested grids covering high resolution regions of the flow. This first AMR structure, called "patchbased AMR", consists of computational-grid building blocks as rectangular patches of various sizes. An alternative method was proposed, the "tree-based" AMR (Khokolov 1998), where the parent cells are refined into children cells on a cell-by-cell basis. These adaptive mesh structures are coupled with gridbased, fluid-dynamics schemes handling high-resolution shock capturing. Nowadays, high order Godunov methods appear to be amongst the best schemes to capture discontinuities within only a few cells (e.g. Teyssier 2002; Matsumoto \& Hanawa 2003; Ziegler 2005; Fromang et al. 2006).

\subsubsection{The RAMSES code}

We use the AMR code RAMSES (Teyssier 2002), which integrates the "tree-based" data structure allowing recursive grid refinements. RAMSES uses a second-order, Godunov hydrodynamical scheme coupled with a gravity solver. Furthermore, it has the possibility to use variable timesteps at each refinement level. Concerning time integration, RAMSES uses a secondorder midpoint scheme, where positions and velocities are updated by a predictor-corrector step. Recently, an MHD version of RAMSES was developed by Fromang et al. (2006).

The Godunov hydrodynamical solver is able to capture discontinuities at a high precision level. The equations solved in RAMSES are the Euler equations in their conservative form

$\frac{\partial \rho}{\partial t}+\nabla \cdot[\rho \boldsymbol{u}]=0$ 
$\frac{\partial \rho \boldsymbol{u}}{\partial t}+\nabla[\rho \boldsymbol{u} \otimes \boldsymbol{u}+P \mathbb{I}]=-\rho \nabla \Phi$,

$\frac{\partial \mathrm{E}}{\partial t}+\nabla[\boldsymbol{u}(E+P)]=-\rho \boldsymbol{u} \cdot \nabla \Phi$

where $P$ is the gas pressure (I is the identity matrix), $\rho$ the density, $\boldsymbol{u}$ the velocity, $E$ the total energy density and $\Phi$ the gravitational potential. The system of equations is closed with the barotropic equation of state (3).

One of the main advantages of solving the Euler equations in their conservative form, is that no energy sink due to numerical errors can alter the flow dynamics (ignoring the source terms due to gravity). Equations (4)-(6) are solved with a Lax-Friedrich Riemann solver, known to be one of the most simple and robust schemes. In Appendix C, we report on the influence of using the Roe solver which is less diffusive but considerably more complex and numerically expensive.

The timestep is determined independently for each refinement level $\ell_{i}$, using standard stability constraints for the hydrodynamical solver. Each level $\ell_{i}$ evolves according to its own timestep.

\subsubsection{Initial conditions for RAMSES}

One practical limitation of AMR codes is the use of Cartesian grids. RAMSES works with a cubic volume, so that a part of the calculation box is lost when we describe a sphere. The outer region of the sphere is also at a temperature of $10 \mathrm{~K}$ but is 100 times less dense. The outer gas has no effect on the dynamics of the sphere because the two parts are well-separated. The sphere radius is equal to a quarter of the box length in order to minimize border effects.

\subsection{SPH: smoothed particles hydrodynamics}

\subsubsection{A brief overview}

$\mathrm{SPH}$ is the most popular fully Lagrangian method used to describe gravitational collapse because of its simplicity for $3 \mathrm{D}$ codes and its versatility to incorporate self-gravity. SPH was first designed to simulate nonaxisymmetric phenomena for astrophysical gases (Lucy 1977; Gingold \& Monaghan 1977). This method is straightforward to use and develop, and efficiently produces robust results. It can also effectively model hydrodynamical flows in regions of very low mass or particle density. It does not need a grid to calculate spatial derivatives, but consists of a set of discrete particles describing the state of the fluid. The spatial derivatives are found by analytical differentiation of interpolation formulae. SPH particle $i$ should not be perceived as a real fluid element, but as a mathematical entity with coordinates $\boldsymbol{r}_{i}$, velocity $\boldsymbol{v}_{i}$, mass $m_{i}$ (i.e. $m$ since all particles have the same mass in the present calculations) and thermal energy $e_{i}$. The evolution of the fluid is determined by following the motion of the particles, under the influence of interparticle forces which represent the effects of pressure, viscosity (see below) and self-gravity.

The main advantage of SPH is its strict Galilean-invariant property and its simplicity. Resolution elements are then concentrated in high-density regions in SPH methods. The standard SPH formalism uses artificial viscosity for the hydrodynamics. Some alternative formalism such as Godunov SPH has been proposed (e.g. Inutsuka 1994) in order to avoid the use of artificial viscosity, but these methods have yet to be well developed and tested. SPH has been used by several authors to study fragmentation (Bonnell 1994; Bate \& Burkert 1997; Goodwin et al. 2004; Hennebelle et al. 2004).

\subsubsection{Hydrodynamical method for DRAGON}

We use the standard SPH code DRAGON (Turner et al. 1995; Goodwin et al. 2004), that is in its most simple version. In standard SPH, the integral interpolant for the variable $A\left(\boldsymbol{r}_{i}\right)$ is approximated by a summation interpolation over the particle's nearest neighbors:

$A_{s}\left(\boldsymbol{r}_{i}\right)=\sum_{j} m_{j} \frac{A\left(\boldsymbol{r}_{j}\right)}{\rho\left(\boldsymbol{r}_{j}\right)} W\left(\left|\boldsymbol{r}_{i}-\boldsymbol{r}_{j}\right|, h_{i j}\right)$,

where $A\left(\boldsymbol{r}_{j}\right)$ is the value associated with particle $j, h_{i j}=\left(h_{i}+\right.$ $\left.h_{j}\right) / 2$, and $h_{i}$ is the adaptive smoothing length of particle $i$, defined such that the particle kernel volume contains a constant mass, i.e. a constant number of neighbors $N_{N}$. The interpolation mass is then given by $m N_{\mathrm{N}}$.

We use a standard artificial viscosity scheme (Gingold \& Monaghan 1983):

$\Pi_{i j}=\left\{\begin{array}{cc}\left(-a_{1} C_{0, i j} \mu_{i j}+a_{2} \mu_{i j}^{2}\right) / \rho_{i j} & \text { if } \boldsymbol{u}_{i j} \cdot \boldsymbol{r}_{i j} \leq 0, \\ 0 & \text { otherwise }\end{array}\right.$

with

$\mu_{i j}=\frac{h_{i j} \boldsymbol{u}_{i j} \cdot \boldsymbol{r}_{i j}}{\boldsymbol{r}_{i j}^{2}+a_{3}^{2}}$

where $\boldsymbol{u}_{i j}=\boldsymbol{u}_{i}-\boldsymbol{u}_{j}, \boldsymbol{r}_{i j}=\boldsymbol{r}_{i}-\boldsymbol{r}_{j}$, and $C_{0, i j}$ and $\rho_{i j}$ denote arithmetic means of the isothermal sound speed and density of the particles $i$ and $j$. The free parameters $a_{1}, a_{2}$ and $a_{3}$ regulate the strength of the viscosity. In our case, we have the combination $a_{1}=1, a_{2}=2$ and $a_{3}=0.1 h_{i j}$. This artificial viscosity has been the subject of a number of discussions in which authors suggest alternative artificial viscosity. One common improvement is to use a time-dependent viscosity (Morris \& Monaghan 1997). We look at the influence of the viscosity scheme in Appendix B.

The gravitational force between a pair of particles obeys a simple inverse-square law, unless the particles are very close. Under this circumstance, the gravity force has to be softened to avoid violent two-body interactions. According to Bate \& Burkert (1997), the gravitational softening length should be the same as the hydrodynamic smoothing length. Calculation of the gravitational acceleration of a particle is speeded up using an octal Spatial Tesselation Tree (STT) (Hernquist 1987). It also accounts for the quadrupole moments of the mass distributions. DRAGON benefits from the implementation of "sink particles" creation (Bate et al. 1995), used to continue the calculations without resolving processes on extremely short time-scales. Last but not least, DRAGON uses multiple-particle timesteps.

Finally, we allow a variation of the number of neighbors $\Delta N_{\mathrm{N}}$ less than $10 \%$ of $N_{\mathrm{N}}$ in our SPH calculations, i.e $\Delta N_{\mathrm{N}}=5$ when $N_{\mathrm{N}}=50$. Attwood et al. (2007) shows that the smaller $\Delta N_{\mathrm{N}}$, the less diffusive is SPH. Ideally, $\Delta N_{N}$ should be set to 0 . We report on the influence of setting $\Delta N_{N}=0$ for local angular momentum conservation in Appendix B.

\subsubsection{Initial conditions for DRAGON}

We apply the method as it was originally presented by Whitworth et al. (1995). We derive an initial particle distribution by allowing hydrodynamical forces to act over a few timesteps to randomly settled particles. Another approach sometimes used is 
to take initial hexagonal-close-paced lattice of SPH particles to generate initial conditions. However, standard SPH calculations start from noisy initial particle distributions at present time (e.g. Arreaga-García et al. 2007). Note that we do not need intercloud and external particles to confine the ones within the sphere since our model is initially far from equilibrium.

\subsection{The Jeans criterion in numerical codes}

\subsubsection{Refinement criterion for the AMR method}

Our refinement criterion is based on the Jeans length resolution which is necessary to accurately treat gravitational collapse. We impose a minimum number of points $N_{\mathrm{J}}$ per Jeans length $\lambda_{\mathrm{J}}$. The cells' dimensions must be smaller than a constant fraction of the local Jeans length. The dimension of cells belonging to the $\ell_{i}$ refinement level is $L_{\mathrm{box}} / 2^{\ell_{i}}$, where $L_{\mathrm{box}}$ is the physical length of the simulation box. The mesh is locally refined in order to satisfy the local Jeans criterion:

$\frac{L_{\mathrm{box}}}{2^{\ell_{i}}}<\frac{\lambda_{\mathrm{J}}}{N_{\mathrm{J}}}$

Truelove et al. (1997) defined a minimum resolution condition for the validity of grid-based simulations aimed at modeling the collapse of a molecular cloud core, namely $N_{\mathrm{J}}>4$. This condition ensures that the collapse is of physical rather than of numerical origin.

\subsubsection{Jeans length description with a SPH code}

In standard SPH, the resolution in mass is fixed and thus the Jeans length resolution deteriorates with increasing density for an isothermal gas. The minimum resolvable mass must then be larger than the interpolation mass. Bate \& Burkert (1997) showed that the behaviour of a Jeans-mass clump of gas with radius $\sim h$ is dominated by the numerical implementation. Bate \& Burkert (1997) take the smallest mass that can be resolved in SPH calculations to be equal to the mass of $\sim 2 N_{\mathrm{N}}$ particles. According to this criterion, we can determine an initial number of SPH particles necessary to solve the Jeans length in the simulations.

The Jeans mass is $M_{\mathrm{J}} \sim 6 G^{-3 / 2} \rho^{-1 / 2} C_{\mathrm{s}}^{3}$ and the minimum resolvable mass is $M_{\text {res }}=m N_{\mathrm{N}}$. Hence, we can define a Jeans condition corresponding to the minimum value of $C_{\mathrm{s}}^{3} \rho^{-1 / 2}$, given by the barotropic equation of state (3), i.e. $2^{3 / 2} C_{0}^{3} \rho_{c}^{-1 / 2}$ :

$m<m_{\max } \sim \frac{2^{3 / 2} 6 C_{0}^{3}}{2 N_{\mathrm{N}} G^{3 / 2} \rho_{\mathrm{c}}^{1 / 2}} \sim \frac{5.35 \times 10^{-3}}{N_{\mathrm{N}}} M_{\odot}$.

Considering an initial spherical mass $M_{0}=1 M_{\odot}$, the initial number of particles $N_{\mathrm{p}}$ has to satisfy $N_{\mathrm{p}}>M_{0} / m_{\max } \sim 9300$ if $N_{\mathrm{N}}=50$. This is the critical number of particles used in SPH calculations to study the collapse of a dense core. We have in that case exactly $2 N_{\mathrm{N}}$ (i.e. two resolution elements) particles per critical Jeans mass. Hubber et al. (2006) shows that with this numerical resolution, standard SPH will capture fragmentation which is genuine and resolved.

As mentioned before, the mass resolution is fixed in standard SPH. In term of Jeans mass, the resolution is therefore high at the beginning of the simulation and decreases when the density increases up to the critical density. It is nevertheless instructive to have a means of comparing the SPH and AMR resolution. We therefore define for the SPH the parameter $N_{\mathrm{J}}$ such that $N_{\mathrm{J}}^{3}=M_{\mathrm{J}} / M_{\text {res }}$ is the number of resolution element per Jeans
Table 1. Summary of the different simulations for the case with no rotation (left table: SPH; right table: AMR). $N_{\mathrm{i}}$ for the AMR calculations gives us the number of cells describing the initial sphere.

\begin{tabular}{|c|c|c|c|c|c|c|c|}
\hline$N_{\mathrm{p}}$ & $N_{\mathrm{N}}$ & $N_{\mathrm{J}}$ & $t_{0}(\mathrm{kyr})$ & & & & \\
\hline $5 \times 10^{3}$ & 50 & 1.86 & 108.6 & $\ell_{\min }$ & $N_{\mathrm{i}}$ & $N_{\mathrm{J}}$ & $t_{0}(\mathrm{kyr})$ \\
\hline $1 \times 10^{4}$ & 50 & 2.33 & 102 & 5 & 2145 & 10 & 109 \\
\hline $5 \times 10^{4}$ & 50 & 4. & 94.1 & 6 & 17160 & 10 & 98 \\
\hline $2 \times 10^{5}$ & 50 & 6.35 & 91.5 & 7 & 137260 & 10 & 95 \\
\hline $5 \times 10^{5}$ & 50 & 8.61 & 90.6 & & & & \\
\hline
\end{tabular}

\begin{tabular}{llll}
$5 \times 10^{5}$ & 50 & 8.61 & 90.6 \\
\hline
\end{tabular}

mass. This number is computed at the critical density $\rho_{\mathrm{c}}$, i.e. the most unfavorable case for the SPH. In the dense core where $\rho>\rho_{\mathrm{c}}$, the parameter $N_{\mathrm{J}}$ enables us to compare the resolution achieved by SPH and AMR, respectively. Using particle splitting refinement in SPH (e.g. Kitsionas \& Whitworth 2002) would improve its resolution. Indeed, particle splitting is an economic way to increase the local resolution and thus to avoid violating the Jeans condition in collapse simulations. However, as mentioned in the introduction, the aim of the present paper is to compare the AMR and the SPH within their standard implementation.

\section{Free-fall time and angular momentum conservation}

We start by comparing the global properties of the collapse in the two codes in the simple case of a uniform-density sphere collapse with no perturbation. We look at the collapse time, the accretion shock and finally the angular momentum conservation.

We completed a first set of simulations using a wide range of resolution parameters. The initial sphere is set up by parameters $\alpha=0.65$ corresponding to an initial radius $R_{0}=9.2 \times 10^{16} \mathrm{~cm}$ and a density $\rho_{0} \sim 6.02 \times 10^{-19} \mathrm{~g} \mathrm{~cm}^{-3}$. The corresponding freefall time is $t_{\mathrm{ff}}=\left(3 \pi / 32 G \rho_{0}\right)^{1 / 2} \sim 86 \mathrm{kyr}$.

\subsection{Free-fall time}

The first step is to compare the calculations collapse time when the initial sphere is not rotating (i.e. $\beta=0$ ). Note that since $\alpha$ is large and since we use a barotropic equation of state, we expect to find a value larger than the free-fall time. Then, we use as a reference time $t_{0} \gtrsim\left(t_{\mathrm{ff}}\right)$ for which $\rho_{\max }=\rho_{c}$. Table 1 gives collapse times $t_{0}$ as a function of resolution parameters for AMR and SPH calculations. AMR calculations were completed for the parameters $N_{\mathrm{J}}=10$ and $\ell_{\min }=5,6$ and 7 . SPH calculations were derived using parameters values $N_{\mathrm{p}}$ ranging from $5 \times 10^{3}$ to $2 \times 10^{5}$ and a number of neighbors $N_{\mathrm{N}}=50$. We note that with $N_{\mathrm{p}}=5 \times 10^{3}$, we do not satisfy the Bate \& Burkert (1997) criterion, but the mass of our resolution element, i.e. the sphere containing the $N_{\mathrm{N}}$ neighbors, is smaller that the critical Jeans mass.

With increasing numerical resolution, the numerical time $t_{0}$ decreases and seems to converge toward a value slightly greater than the free-fall time. Time $t_{0}$ changes by less than $5 \%$ between AMR calculations with $\ell_{\min }=6$ and 7 , and SPH calculations with $N_{\mathrm{p}}=5 \times 10^{4}$ and $2 \times 10^{5}$. Dynamical times $t_{0}$ in $\mathrm{SPH}$ calculations are closer to the free-fall time than the AMR ones. This is partly due to the higher initial resolution in SPH. 
B. Commerçon et al.: Protostellar collapse: a comparison between SPH and AMR calculations
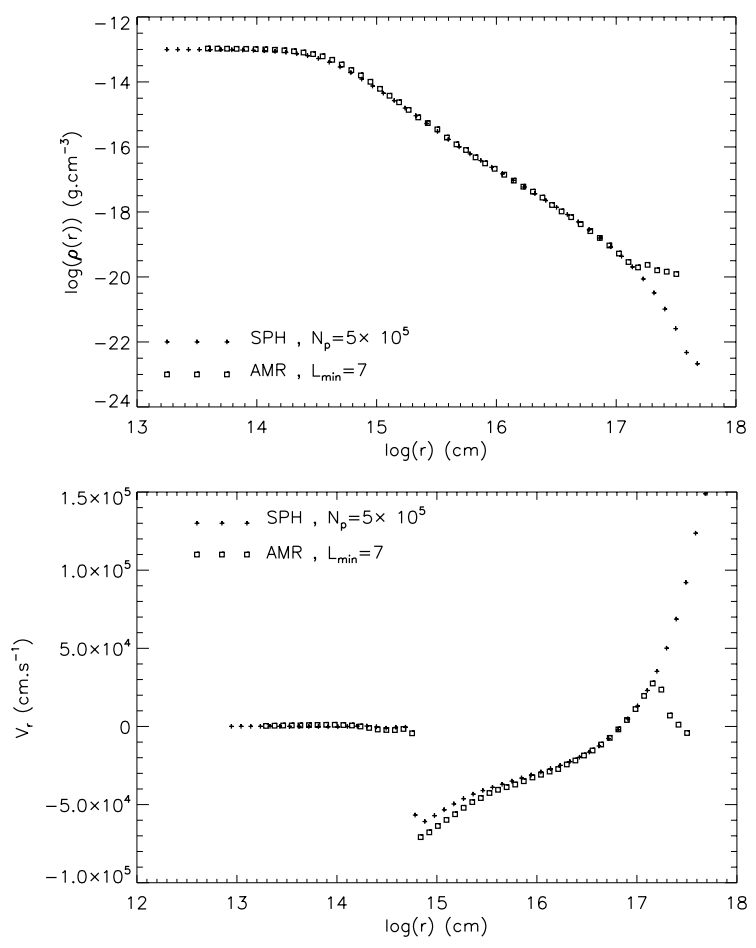

Fig. 1. a) Density profiles at $t_{0}$ as a function of the radius for the case with $\beta=0.01$ and for the two most resolved simulations, namely $\ell_{\min }=$ $7, N_{\mathrm{J}}=10$ for the AMR (squares) and $N_{\mathrm{p}}=5 \times 10^{5}, N_{\mathrm{N}}=50$ for the SPH (crosses). b) Illustration of the accretion shock: radial velocity profiles for the two most resolved AMR and SPH simulations in the $x y$-plane at $\sim t_{0}+1.2 \mathrm{kyr}$.

\subsubsection{Collapse and accretion shock with rotation $\beta=0.01$}

The gas sphere is now in solid rotation about the $z$-axis. We set $\beta=0.01$, corresponding to an orbital time $t_{\text {rot }}=2.8 \times 10^{3} \mathrm{kyr}$. Table 2 summarizes the different SPH and AMR calculations executed for this case. In order to illustrate the core resolution, we provide the quantity $N_{\text {core }}$ representing the number of cells/particles with density $\rho>1 \times 10^{-15} \mathrm{~g} \mathrm{~cm}^{-3}$ at $t_{0}$. AMR simulations have been performed with different minimum refinement levels $\ell_{\min }$ ranging from 5 to 7 and a refinement criterion $N_{\mathrm{J}}$ ranging from 4 to 10 . As expected, at a constant $N_{\mathrm{J}}$, the various AMR calculations show a convergence. The SPH simulations were performed with a constant number of neighbors $N_{\mathrm{N}}=50$, and a total number of particles $N_{\mathrm{p}}$ ranging from $5 \times 10^{3}$ to $5 \times 10^{5}$. For standard SPH, the effects of the decrease in resolution with increasing density are demonstrated by the fact that, for equivalent initial conditions, it is easier to achieve a better core resolution using AMR.

In Fig. 1a we show density profiles as a function of the radius in the equatorial plane for AMR calculations with $\ell_{\min }=7$ and $N_{\mathrm{J}}=10$, and for SPH with $N_{\mathrm{p}}=5 \times 10^{5}$ and $N_{\mathrm{N}}=50$. The density profiles are very similar, indicating good convergence between the two methods. The behaviour differs at relatively high radius because of the external gas in the AMR method. In the present simulations, the dynamical time to reach $\rho_{\mathrm{c}}$ is increased by $\sim 5 \mathrm{kyr}$, because of the rotational support. As seen in Table 2 , when one increases the resolution, one seems to converge toward this time.

When the core becomes adiabatic, the angular momentum conservation induces the formation of an accretion disk around the central object. The centrifugal force becomes comparable to the gravitational one on the equatorial plane, slowing down the
Table 2. Summary of the different simulations (upper table: SPH, lower table: AMR) performed to study angular momentum conservation.

\begin{tabular}{ccccc}
\hline \hline$N_{\mathrm{p}}$ & $N_{\mathrm{N}}$ & $N_{\mathrm{J}}$ & $N_{\text {core }}$ & $t_{0}(\mathrm{kyr})$ \\
\hline $5 \times 10^{3}$ & 50 & 1.86 & 225 & 115 \\
$1 \times 10^{4}$ & 50 & 2.34 & 422 & 107 \\
$5 \times 10^{4}$ & 50 & 4. & 1833 & 98 \\
$2 \times 10^{5}$ & 50 & 6.35 & 7055 & 95 \\
$5 \times 10^{5}$ & 50 & 8.61 & 17309 & 93 \\
\hline
\end{tabular}

\begin{tabular}{cccccc}
\hline \hline$\ell_{\min }$ & $N_{\mathrm{i}}$ & $N_{\mathrm{J}}$ & $N_{\text {core }}$ & Tot. cells & $t_{0}(\mathrm{kyr})$ \\
\hline 5 & 2145 & 6 & 3928 & $\sim 9.1 \times 10^{4}$ & 150 \\
5 & 2145 & 10 & 30752 & $\sim 1.6 \times 10^{5}$ & 116 \\
6 & 17160 & 4 & 4016 & $\sim 3.1 \times 10^{5}$ & 116 \\
6 & 17160 & 10 & 28800 & $\sim 3.7 \times 10^{5}$ & 109 \\
7 & 137260 & 10 & 29944 & $\sim 2.2 \times 10^{6}$ & 96 \\
\hline
\end{tabular}

collapse. The outer collapsing gas, which has a supersonic infalling speed, suddenly meets the static gas of the core, creating an accretion shock. This shock can be clearly seen in Fig. 1b where the radial velocity component averaged over the equatorial plane is displayed. The accretion shock is described slightly more accurately with the AMR method. The SPH curve is smoother before the shock due to the artificial viscosity scheme. The slope before the shock strongly depends on the hydrodynamical solver so the results illustrate the difference between the hydrodynamical methods used in our two codes.

\subsection{Theoretical local angular momentum}

We now investigate the issue of angular momentum conservation. We note that the equations for both SPH and AMR equations imply that linear momentum is conserved. Considering our axisymmetric model, without azimuthal perturbation, we can easily investigate the effect of numerical resolution on angular momentum conservation. The local angular momentum should be well conserved, until azimuthal symmetry is broken. The loss of local angular momentum in our model is only due to unphysical transport inherent to the numerical methods used in the two codes. Due to their Lagrangian properties, the SPH calculations can provide a measure of the initial angular momentum of each particle, that is the angular momentum that the particle should have if angular momentum is perfectly conserved. Having access to the particle initial angular momentum, the loss of angular momentum is easily calculated. The azimuthal velocity component, directly linked to the angular momentum $\boldsymbol{J}$, is given by

$v_{\theta}=\frac{x v_{y}-y v_{x}}{r}$

where $r$ is the distance from the rotation axis

$r=\sqrt{x^{2}+y^{2}}$

and the angular momentum

$\boldsymbol{J}=x v_{y}-y v_{x}$

The angular momentum conservation along the $z$-axis allows us to write

$\boldsymbol{J}=x v_{y}-y v_{x}=x_{0} v_{y, 0}-y_{0} v_{x, 0}=\boldsymbol{J}_{0}$. 


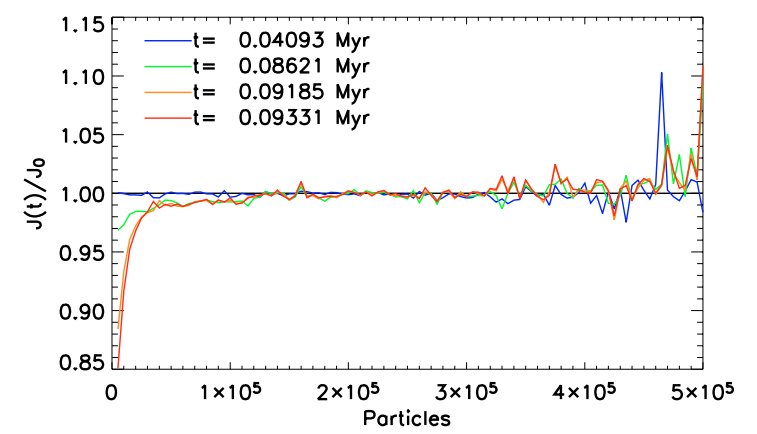

Fig. 2. Ratio between the angular momentum $\boldsymbol{J}(t)$ over the initial angular momentum $\boldsymbol{J}_{0}$ at different times for the SPH simulation with $N_{\mathrm{p}}=5 \times 10^{5}$ and $N_{\mathrm{N}}=50$. The ratio is plotted as a function of the number of particles, ordered in decreasing density. The value of the ratio is a mean value over 7500 particles. The red curve corresponds to results at $t_{0}$.

Thus, the theoretical angular velocity of a particle at time $t$ is determined by the ratio between its initial angular momentum $\boldsymbol{J}_{0}=\left(x_{0} v_{y, 0}-y_{0} v_{x, 0}\right)$ and its actual radius:

$v_{\theta, \mathrm{th}}=\frac{\boldsymbol{J}_{0}}{r}$.

Using Eq. (16), we compare the theoretical angular velocity component to their numerical values, and in particular with the AMR results for which we do not have access to a theoretical value. Since SPH and AMR density profiles are almost identical at $t_{0}$, we suppose that the previous mapping giving $\boldsymbol{J}_{0}\left(r_{0}\right)$ as a function of the radius in the SPH runs is also valid for the AMR calculations. Note also that the method cannot account for the displacement of the particles that would arise by changing the angular momentum.

\subsubsection{Azimuthal velocity component}

Figure 2 presents the ratio of the angular momenta $\boldsymbol{J}(t)$ and $\boldsymbol{J}_{0}$ for the SPH simulation with $N_{\mathrm{p}}=5 \times 10^{5}$ and $N_{\mathrm{N}}=50$. The particles are arranged in order of decreasing density and the ratio is averaged over 7500 particles. A first interesting result is that denser particles lose more angular momentum. At $t_{\mathrm{ff}}$, denser particles have lost $3 \%$ of their initial angular momentum. In less resolved calculations, i.e. $N_{\mathrm{p}}=5 \times 10^{3}$ particles, the effect is stronger and the densest ones lose more than $10 \%$ of their initial angular momentum in a free-fall time $t_{\mathrm{ff}}$. This percentage slightly decreases when the number of particles is increased. The reason is that the denser the particle the larger the viscous torque (see below) and thus the larger the angular momentum transport. This numerical transport is amplified when the core is close to becoming adiabatic.

Figure 3 shows the cumulated hydrodynamical (left-hand side) and viscous (right-hand side) torques on the rotational axis for the same SPH calculations at the same times as in Fig. 2. In principle, there should not be any torque on the rotational axis because of this axisymmetric model. The cumulated torques are computed and summed at each timestep for each particle. The value plotted is an average over 7500 particles and particles are ordered in decreasing density. Denser particles have the largest cumulated hydrodynamical and viscous torques. The friction forces corresponding to the hydrodynamical torque are stronger for these particles, which is due to the strong differential velocity.
Figure 4a displays the azimuthal velocity component as a function of the radius $r$ on the $x y$-plane for the SPH simulations. The theoretical azimuthal velocity profile (solid line) is obtained following the previous section. It is obvious that low resolution simulations are not able to conserve properly the angular momentum. With $5 \times 10^{3}$ particles, we obtain counter-rotating particles at the center (not illustrated in Fig. 4a because we make the average of the angular velocity in the $x y$-plane that smoothes the profiles). It appears that a minimum of $5 \times 10^{4}$ particles is required to maintain angular momentum loss within less than $10 \%$, for the case of the present study. The improvement of angular momentum conservation eventually saturates for large numbers of particles. We checked that using a larger number of neighbors does not improve the conservation of local angular momentum.

Figure $4 \mathrm{~b}$ presents results obtained using the RAMSES code. AMR curves are plotted and compared with the theoretical results obtained previously using SPH. The simulations with $\ell_{\text {min }}=6$ and $\ell_{\text {min }}=7$ are close to the theoretical curve. In both AMR simulations with $N_{\mathrm{J}}=10$, dense core resolution is higher than with SPH. For an initial resolution of $\ell_{\min }=5$, the AMR scheme does produce some angular momentum lag. This can be due to the fact that with a poor initial resolution, the interpolation of the gravitational potential tends to convert gravitational energy into rotational energy. The outer gas does not alter the angular momentum conservation for AMR calculations because of its tiny density.

In Figs. 5a, b, we plot the integrated mass and the ratio of numerical over theoretical angular momentum for AMR calculations with $\ell_{\min }=6, N_{\mathrm{J}}=10$ and for SPH calculations with $N_{\mathrm{p}}=5 \times 10^{5}, N_{\mathrm{N}}=50$. In Fig. 5a, most of the mass of the forming disk remains within a radius $\sim 1 \times 10^{16} \mathrm{~cm}$, for which AMR and SPH have divergent results. In Fig. 5b, angular momentum is clearly transported to the outer regions with SPH, but has a trend to be transported to the inner regions with AMR. The overall angular momentum is well conserved in both calculations, but local properties seem to be affected by initial resolution. SPH resolution is much better at the beginning of the calculations, which enables SPH to properly conserve local angular momentum at $r>1.4 \times 10^{15} \mathrm{~cm}$ at $t_{0}$. In contrast, the smaller initial resolution of the sphere for AMR implies that angular momentum is conserved far less in this region. This effect reverses at lower radius where AMR can reach smaller scales contrary to standard SPH.

The density profiles obtained with the two methods converge towards a similar solution (cf. Fig. 1a), but a closer analysis of the velocity profiles shows discrepancies. The angular velocity profiles appear to imply that local angular momentum is better conserved by the AMR method than with the SPH method, for the code implementation that we have used. Both can be improved using appropriate methods (see Appendices B and C).

\section{Fragmentation}

\subsection{Model}

Prestellar core fragmentation is a highly non-linear process. A key issue is to understand the extent to which the fragmentation that occurs in numerical simulations, is influenced by the numerical scheme and resolution.

To study dense core fragmentation, we choose the same previous spherical model and impose a $m=2$ azimuthal density perturbation:

$\rho(\theta)=\rho_{0}[1+A \cos (m \theta)]$, 

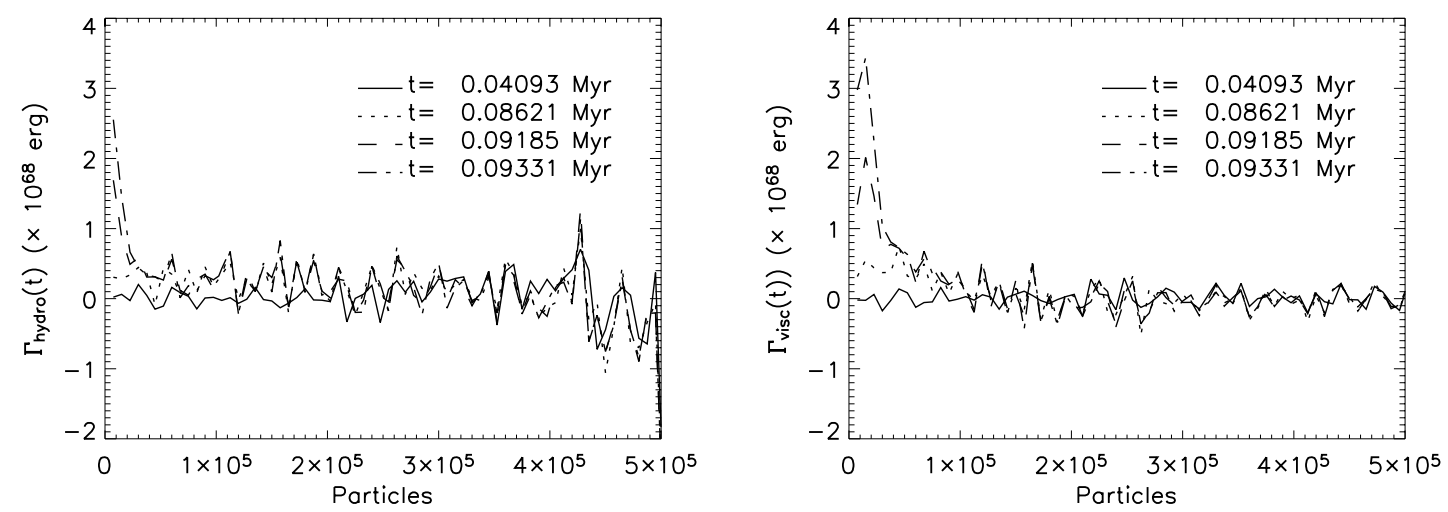

Fig. 3. Left plot: cumulated hydrodynamical torque on the rotational axis for SPH calculations with $N_{\mathrm{p}}=5 \times 10^{5}$ and $N_{\mathrm{N}}=50$ and at the same times as in Fig. 2. Right plot: cumulated torque on the rotational axis due to the standard artificial viscosity for the same calculations and times. Particles are ordered in decreasing density and the torques are averaged over 7500 particles. At $t_{0}$ the cumulated torques are significant for the densest particles.
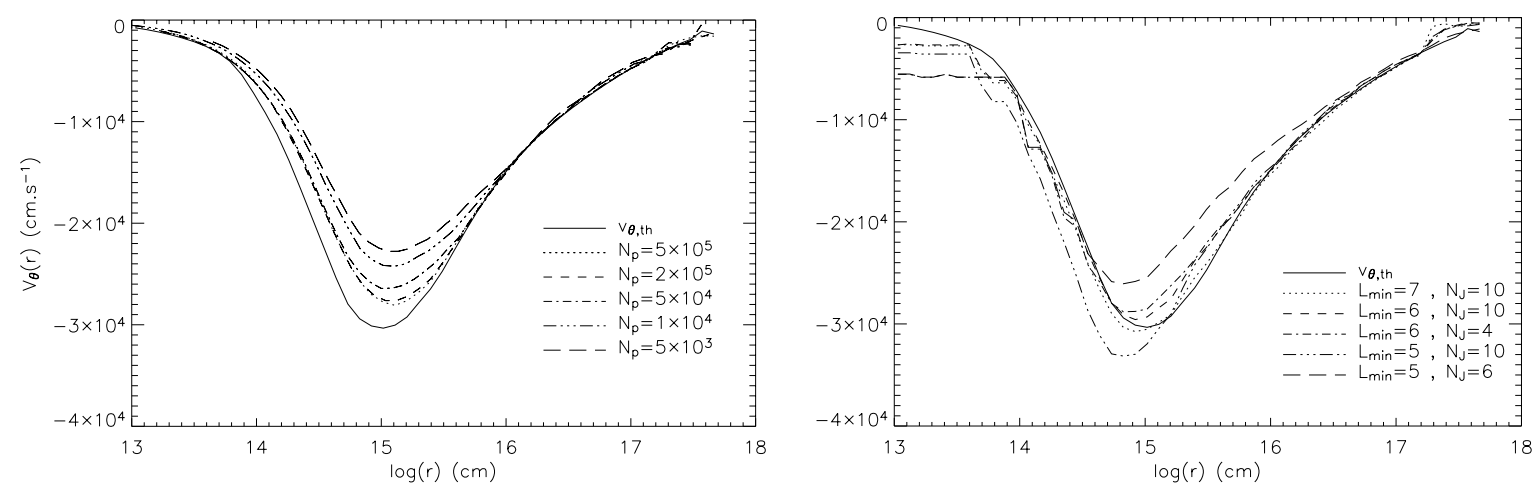

Fig. 4. Azimuthal velocity as a function of the radius on the equatorial plane for SPH (left) and AMR (right) calculations at corresponding $t_{0}$. The left-hand plot (Fig. 4a) shows SPH results with various $N_{\mathrm{p}}$ and $N_{\mathrm{N}}=50$. The solid line represents the theoretical azimuthal velocity interpolated at $t_{0}$ and is denoted as $v_{\theta, \text { th }}$. The right-hand plot (Fig. $4 \mathrm{~b}$ ) shows AMR results with $N_{\mathrm{J}}=10$ and $\ell_{\min }=5,6$ and 7 . The theoretical azimuthal velocity is plotted also for easy comparison with the SPH results.

where $\rho_{0}$ is the mean sphere density, $A$ the perturbation amplitude and $\theta$ the azimuthal angle in cylindrical coordinates.

The initial conditions are easy to implement for the AMR calculations. The SPH sinusoidal density perturbation is imposed by adjusting the unperturbed $\theta$-coordinate of each particle to a perturbed value $\theta^{*}$ given by:

$\theta=\theta^{*}+\frac{A \sin \left(m \theta^{*}\right)}{m}$.

We compare simulations for three different thermal supports, namely $\alpha=0.35,0.5$ and 0.65 , with a fixed rotational support $\beta=0.04$. In the following, clumps for which the gas density satisfies $\rho>1 \times 10^{-12} \mathrm{~g} \mathrm{~cm}^{-3}$ are referred to as fragments.

\subsection{Results for a critical case: $\alpha=0.5, \beta=0.04$ and $A=0.1$}

We now consider the effects of varying the numerical parameters. First, we study the effect of varying the initial grid resolution $\ell_{\min }$ and the number of cells within a Jeans length $N_{\mathrm{J}}$ for AMR calculations. Then, we present our SPH calculations with various number of neighbors $N_{\mathrm{N}}$ and of particles $N_{\mathrm{p}}$. For this set of calculations, the initial parameters are: $\rho_{0}=1.35 \times$ $10^{-18} \mathrm{~g} \mathrm{~cm}^{-3}, R_{0}=7.07 \times 10^{16} \mathrm{~cm}, \Omega_{0}=2.12 \times 10^{-13} \mathrm{rad} \mathrm{s}^{-1}$ and $t_{\mathrm{ff}}=57 \mathrm{kyr}$. The initial perturbation amplitude is $A=0.1$.
Table 3 summarizes the calculations we performed for this case. Informations about the core resolution (i.e. $\rho>1 \times$ $10^{-15} \mathrm{~g} \mathrm{~cm}^{-3}$ ) and the total number of cells are given at $t=$ $t_{0}+4 \mathrm{kyr}$. The dynamical times to reach the collapse are quite similar, within less than $2 \%$. Synchronizing calculations at $t_{0}$ is then well justified. In the following sections, we consider core evolutions over a few thousand years $\left(\sim 10 \%\right.$ of $\left.t_{0}\right)$.

\subsubsection{Detailed study of the effect of $N_{\mathrm{J}}$ and $\ell_{\min }$ for AMR calculations}

In Fig. 6, we show density maps on the equatorial plane at four different timesteps for AMR calculations with, from left to right, $\ell_{\min }=6$ and $N_{\mathrm{J}}=4,10$ and 15 and $\ell_{\min }=7$ (giving $128^{3}$ cells initially) and $N_{\mathrm{J}}=10$. Maps are given, from top to bottom, at $t=t_{0}+4 \mathrm{kyr}, t=t_{0}+5 \mathrm{kyr}, t=t_{0}+6 \mathrm{kyr}$, and $t=t_{0}+7 \mathrm{kyr}$. As shown in Fig. 6, fulfilling the Truelove condition (Truelove et al. 1997), $N_{\mathrm{J}}>4$, does not guarantee an accurate fragmentation timescale. The calculations with $N_{\mathrm{J}}=4$ fragments at $t_{0}+5 \mathrm{kyr}$ while other calculations do not fragment until $t=t_{0}+7 \mathrm{kyr}$. This suggests that calculation with $\ell_{\min }=6$ and $N_{\mathrm{J}}=4$ suffers from inaccurate fragmentation, but this latter is inhibited when fragments fall onto the central object before $t_{0}+6 \mathrm{kyr}$. The core will eventually refragment but not at the same time as the other 

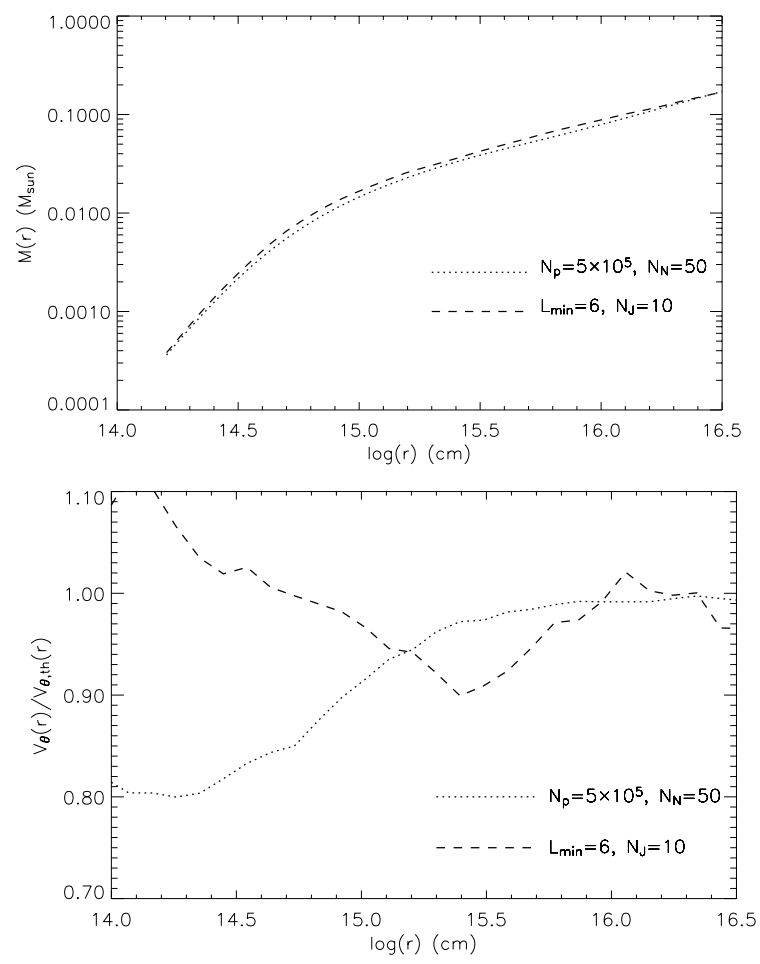

Fig. 5. a) Total integrated mass in the equatorial plane at $t_{0}$ as a function of the radius for AMR calculations with $\ell_{\min }=6, N_{\mathrm{J}}=10$ (dashed-line) and for SPH calculations with $N_{\mathrm{p}}=5 \times 10^{5}, N_{\mathrm{N}}=50$ (dotted line). b) ratio between numerical and theoretical azimuthal velocities at $t_{0}$ as a function of the radius in the equatorial plane for the same calculations.

Table 3. Summary of the different simulations performed with $\alpha=0.5$ and $\beta=0.04$. $N_{\text {core }}$ corresponds to particles/cells whose density satisfies $\rho>1 \times 10^{-15} \mathrm{~g} \mathrm{~cm}^{-3}$ at $t=t_{0}+4 \mathrm{kyr}$. The upper table (Table 3a) gives a summary of the SPH calculations. The lower Table (Table 3b) shows a summary of AMR calculations.

\begin{tabular}{cccc}
\hline \hline$N_{\mathrm{p}}$ & $N_{\mathrm{N}}$ & $N_{\text {core }}$ & $t_{0}(\mathrm{kyr})$ \\
\hline $5 \times 10^{4}$ & 50 & 6997 & 64 \\
$2 \times 10^{5}$ & 50 & 28516 & 63 \\
$5 \times 10^{5}$ & 30 & 72417 & 63 \\
$5 \times 10^{5}$ & 50 & 71804 & 62 \\
$5 \times 10^{5}$ & 100 & 76390 & 63 \\
$5 \times 10^{5}$ & 200 & 71940 & 64 \\
\hline
\end{tabular}

\begin{tabular}{ccccc}
\hline \hline$\ell_{\min }$ & $N_{\mathrm{J}}$ & $N_{\text {core }}$ & Tot. cells & $t_{0}(\mathrm{kyr})$ \\
\hline 6 & 4 & 48680 & $\sim 4.4 \times 10^{5}$ & 66 \\
6 & 10 & 156588 & $\sim 6.2 \times 10^{5}$ & 65 \\
6 & 15 & 263304 & $\sim 7.9 \times 10^{5}$ & 67 \\
7 & 10 & 117108 & $\sim 2.4 \times 10^{6}$ & 65 \\
7 & 15 & 305896 & $\sim 2.7 \times 10^{6}$ & 65 \\
\hline
\end{tabular}

calculations (i.e. $t \gg t_{0}+7 \mathrm{kyr}$ ). With increasing $N_{\mathrm{J}}$, we converge to a fragmented pattern with one central object and two satellites.

Another aspect to be considered quite carefully is the choice of $\ell_{\min }$, i.e. the initial description of the sphere. The results of the two calculations for $N_{\mathrm{J}}=15$, with first $\ell_{\min }=6$ and secondly $\ell_{\text {min }}=7$ are very similar, suggesting that numerical convergence has been achieved. We remark that small differences are apparent in the detailed structures. The satellites formed with $\ell_{\min }=7$ are more structured and compact than those formed with the initial resolution $\ell_{\min }=6$. According to these calculations, fragmentation into two identical satellites and a central object should occur around $t=t_{0}+7 \mathrm{kyr}$.

\subsubsection{Detailed study of the effect of $N_{p}$ and $N_{N}$ for SPH calculations}

We performed a series of SPH calculations with different $N_{\mathrm{p}}$, but with a constant $N_{\mathrm{N}}=50$, and then with a larger $N_{\mathrm{N}}$ for one value of $N_{\mathrm{p}}$.

Figure 7 shows density maps in the equatorial plane for these calculations at four different timesteps, namely, from top to bottom: $t=t_{0}+4 \mathrm{kyr}, t=t_{0}+5 \mathrm{kyr}, t=t_{0}+6 \mathrm{kyr}$ and $t=t_{0}+7 \mathrm{kyr}$. The calculations with $N_{\mathrm{p}}=2 \times 10^{5}$ and $N_{\mathrm{p}}=5 \times 10^{5}$ show good agreement at least until $t_{0}+6 \mathrm{kyr}$ and differ noticeably from the less resolved calculations $\left(N_{\mathrm{N}}=50, N_{\mathrm{p}}=5 \times 10^{4}\right)$ where fragmentation occurs earlier. The dense core fragments in any case, but its fragmentation is delayed when $N_{\mathrm{p}}$ increases (e.g. Nelson 2006). Early fragmentation is here clearly due to a lack of resolution. As shown in Sect. 4.2.1, the conservation of local angular momentum is bad when $N_{\mathrm{p}}$ is low and leads to very inaccurate collapse and fragmentation timescales of the cloud. Once symmetry is broken, it is useless to continue the simulations, since calculations would obviously diverge. This symmetry-breaking occurs earlier in the SPH calculations, first because of the numerical noise inherent to the relaxed, and random, initial particle distributions, and because of the lower resolution of the Jeans length in the disk.

The other fundamental parameter in SPH calculations is the number of neighbors that determine the kernel size. Increasing the number of SPH particles increases the resolution but also introduces numerical noise on smaller scales. The natural way to reduce this noise is to increase the smoothing kernel length, by increasing $N_{\mathrm{N}}$. The effects of varying $N_{\mathrm{N}}$ have been investigated by Lombardi et al. (1999) and Rasio (1999). In particular, Rasio (1999) derived the following results:

- higher accuracy is reached when both $N_{\mathrm{p}}$ and $N_{\mathrm{N}}$ are increased, with $N_{\mathrm{p}}$ increasing faster than $N_{\mathrm{N}}$ so that the smoothing length decreases. One possible scaling (Lombardi et al. 1999) is $N_{\mathrm{N}} \propto N_{\mathrm{p}}^{q}$ with $0.2 \leq q<1$;

- the SPH scheme is consistent in the limit where $\left(N_{\mathrm{N}}, N_{\mathrm{p}}\right) \rightarrow$ $\infty$ and $h \rightarrow 0$;

- convergence (e.g. the number of timesteps) is accelerated by increasing the smoothness of the kernel.

The usual number of neighbors in previous studies is about 50 . However, no study has accurately explored the role played by $N_{\mathrm{N}}$ in the context of star formation. Hence, we performed calculations with a constant $N_{\mathrm{p}}$ and different values of $N_{\mathrm{N}}$. This is illustrated in the right column in Fig. 7 where we report maps of calculations with $N_{\mathrm{p}}=5 \times 10^{5}$ and $N_{\mathrm{N}}=100$. The core has already fragmented at $t_{0}+6 \mathrm{kyr}$ whereas with $N_{\mathrm{N}}=50$ it fragments later. The calculations with parameter set $N_{\mathrm{p}}=2 \times 10^{5}$ and $N_{\mathrm{N}}=50$ is very similar to the later of similar ratio $N_{\mathrm{p}} / N_{\mathrm{N}}$. Other calculations with various $N_{\mathrm{N}}$ are reported in Appendix A. It appears clearly that the greater $N_{\mathrm{N}}$ the earlier fragmentation occurs, because increasing $N_{\mathrm{N}}$ for a fixed $N_{\mathrm{p}}$ decreases the spatial resolution ( $h$ increases).

\subsubsection{Comparison and convergence}

In the previous sections, we show that AMR and SPH calculations converged separately. We now compare the converged calculations. Figure 8 shows density maps in the equatorial plane 

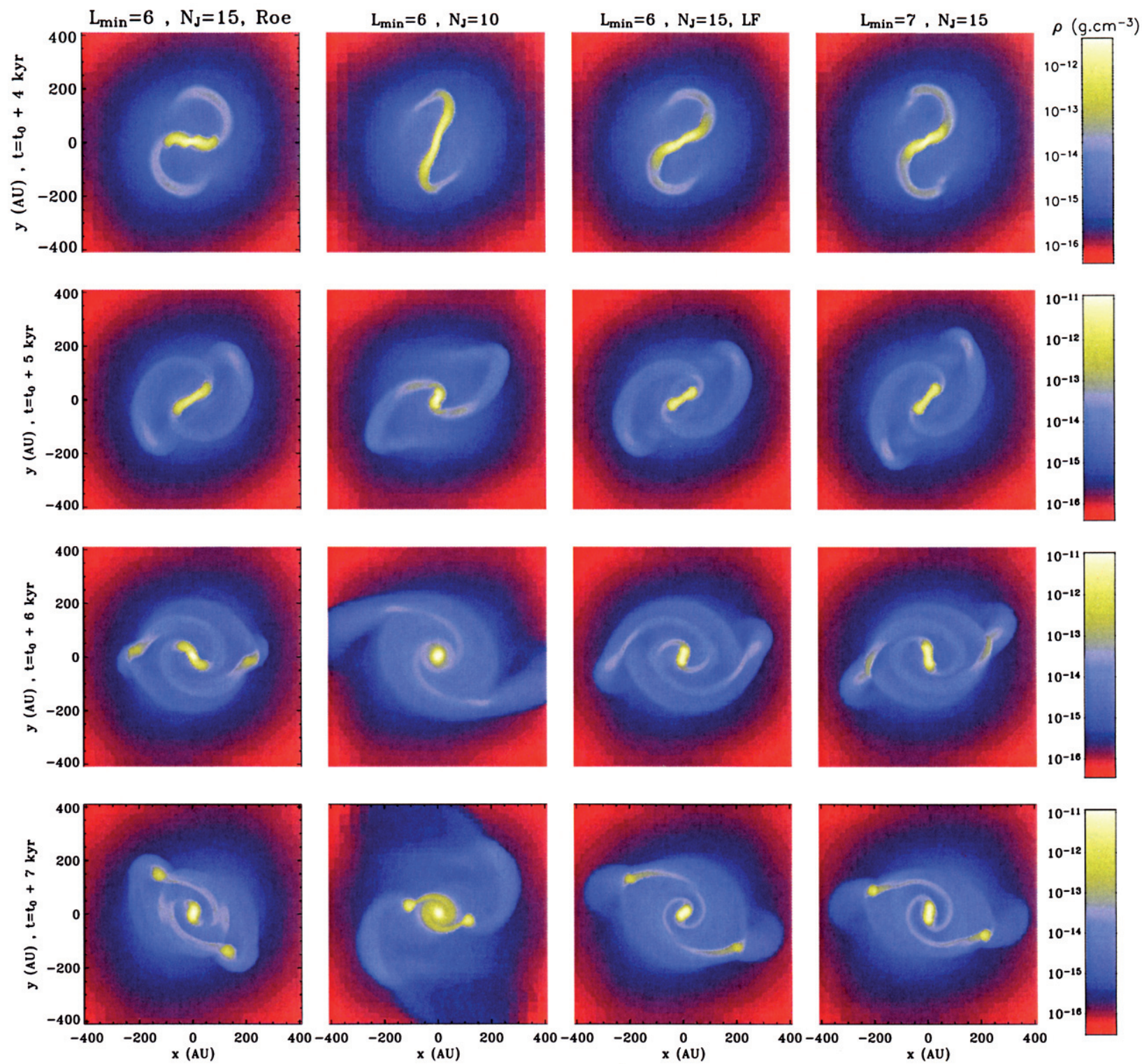

Fig. 6. AMR calculations density maps on the $x y$-plane for the case $\alpha=0.50, \beta=0.04$. From top to bottom, four different times are showed: $t=t_{0}+4 \mathrm{kyr}, t=t_{0}+5 \mathrm{kyr}, t=t_{0}+6 \mathrm{kyr}$ and $t=t_{0}+7 \mathrm{kyr}$. The AMR calculations have been performed with, from left to right columns, $\ell_{\min }=6$ and $N_{\mathrm{J}}=4, N_{\mathrm{J}}=10, N_{\mathrm{J}}=15$ and $\ell_{\min }=7$ and $N_{\mathrm{J}}=15$.

for the results of two of the most resolved calculations at three timesteps, namely, from top to bottom, $t_{0}+5 \mathrm{kyr}, t_{0}+6 \mathrm{kyr}$ and $t_{0}+7$ kyr. The left column shows maps for AMR calculations with $\ell_{\min }=7$ and $N_{\mathrm{J}}=15$ whereas the right column displays SPH maps for calculations with $N_{\mathrm{p}}=5 \times 10^{5}$ and $N_{\mathrm{N}}=50$. We display again the results of Figs. 6 and 7 to illustrate clearly the convergence. Agreement between the two methods for these physical and numerical parameters set is striking for the two first timesteps. The calculations provide the same fragmentation time and pattern, although satellites and the central object are larger for SPH.

Figure 9 shows disk density profiles as a function of the radius averaged in the equatorial plane for the same SPH and AMR calculations. This plot complements Fig. 8 with the last density maps, where fragments are well-developed. Density profiles show a peak at a radius corresponding to satellite positions in the map. Satellite fragments are denser in the SPH calculations, and the central object is less dense and larger compared with the dense elongated shape obtained with the AMR.
Although there are some obvious differences between the two methods, there seems to be a real convergence between the two types of calculations. For the specific case under study, we find a good agreement between AMR calculations with $\ell_{\min }=7$ or 6 , and $N_{\mathrm{J}}=15$ and SPH calculations with $N_{\mathrm{p}}=5 \times 10^{5}$ and $N_{\mathrm{N}}=50$. However, even for the most resolved simulations, the results between the two methods diverge after some time (i.e. $t_{0}+7 \mathrm{kyr}$ for this specific case). This is not surprising because the dynamics becomes non-linear and chaotic, as the initial and numerical noise become amplified.

\subsection{Results for low and high thermal support}

\subsubsection{Results for a least prone to fragment case: $\alpha=0.65$, $\beta=0.04$}

This second series of calculations is the least prone to fragmentation because of its strong thermal support. We use a perturbation 

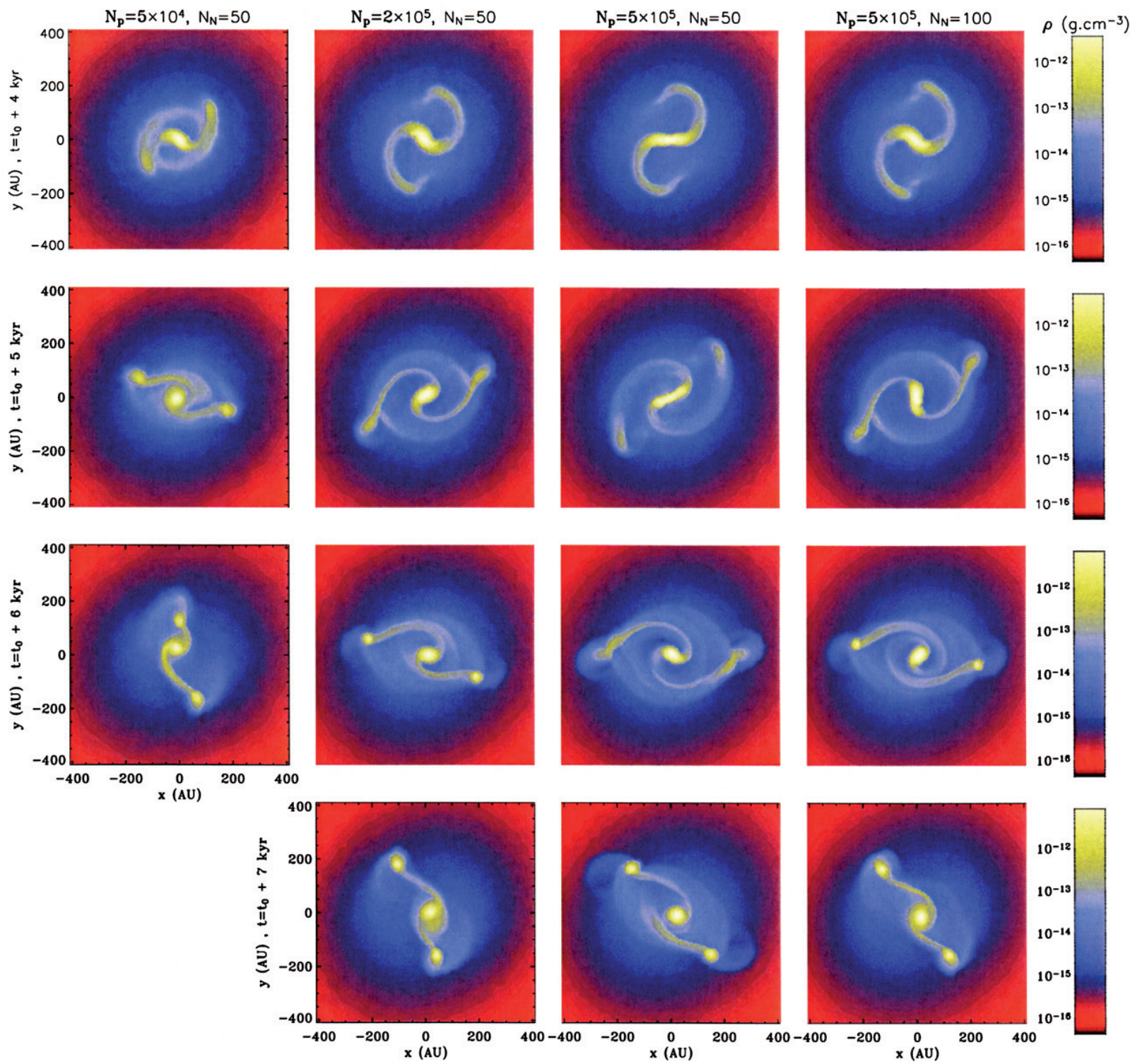

Fig. 7. SPH calculations density maps in the $x y$-plane for the case $\alpha=0.50, \beta=0.04$. From top to bottom, four different times are showed: $t=t_{0}+4 \mathrm{kyr}, t=t_{0}+5 \mathrm{kyr}, t=t_{0}+6 \mathrm{kyr}$ and $t=t_{0}+7 \mathrm{kyr}$. The calculations have been performed with, from left to right, $N_{\mathrm{N}}=50$ and $N_{\mathrm{p}}=5 \times 10^{4}, N_{\mathrm{p}}=2 \times 10^{5}$ and $N_{\mathrm{p}}=5 \times 10^{5}$ and $N_{\mathrm{N}}=100$ and $N_{\mathrm{p}}=5 \times 10^{5}$.

amplitude $A=0.5$ in order to make fragmentation easier if it should occur.

Figure 10 gives density slices on the equatorial plane at $t=t_{0}+10$ kyr. On the left-hand side, we show AMR results for an initial sphere described with $\ell_{\min }=6$ and, from top to bottom, $N_{\mathrm{J}}=4,10$ and 15 . The right-hand column shows slices of SPH calculations with $N_{\mathrm{N}}=50$ and $N_{\mathrm{p}}=5 \times 10^{4}, 2 \times 10^{5}$ and $5 \times 10^{5}$, from top to bottom. In the previous cases, the core has already fragmented into three clumps at this time. In this case, the cloud develops spiral arms with no fragmentation. The cloud fragments in some cases after $t_{0}+50 \mathrm{kyr}$.

AMR and SPH calculations converge quickly to a pattern with only spiral arms and the formation of a single central object when resolution is sufficient.

\subsubsection{Early fragmentation case: $\alpha=0.35, \beta=0.04$}

This last case is the most prone to fragmentation because of its small thermal support against gravitational energy. In this set of calculations, the initial parameters are: $\rho_{0}=3.92 \times 10^{-18} \mathrm{~g} \mathrm{~cm}^{-3}$, $R_{0}=4.95 \times 10^{16} \mathrm{~cm}, \Omega_{0}=3.63 \times 10^{-13} \mathrm{rad} \mathrm{s}^{-1}$ and $t_{\mathrm{ff}}=$ $1.06 \times 10^{12} \mathrm{~s}(\sim 33.6 \mathrm{kyr})$. The initial perturbation amplitude is $A=0.1$. We performed SPH calculations with $N_{\mathrm{p}}$ ranging from $5 \times 10^{4}$ to $5 \times 10^{5}$ and $N_{\mathrm{N}}=50$. The AMR calculations were performed with $\ell_{\min }=6$ and 7 , and $N_{\mathrm{J}}$ varying between 4 and 15 . Although all results are very similar at $t_{0}$, we find some differences at $t_{0}+1 \mathrm{kyr}$. For example, it appears quite clear that AMR calculations with $\ell_{\min }=6$ and $N_{\mathrm{J}}=4$ diverge from the other AMR calculations (tiny spiral arms).

Figure 11 shows density maps in the equatorial plane for the most relevant calculations at $t=t_{0}+3 \mathrm{kyr}$. On the left column, we provide the AMR results with increasing resolution parameter $N_{\mathrm{J}}$ from top to bottom and a constant $\ell_{\min }=6$. According to our previous results, an initial computational domain with $\ell_{\min }=6$ is sufficient to reach convergence for the AMR calculations. The right column shows SPH calculations, with $N_{\mathrm{p}}$ ranging from $5 \times 10^{4}$ to $5 \times 10^{5}$. We seem to reach a convergence between the AMR calculations with $\ell_{\min }=6$ and $N_{\mathrm{J}}>6$. The 


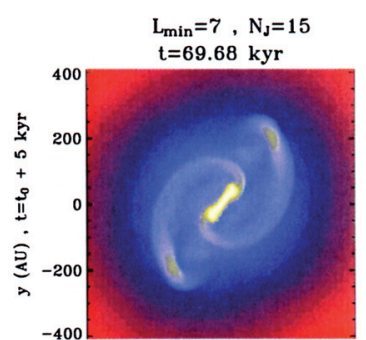

$\mathrm{t}=70.77 \mathrm{kyr}$

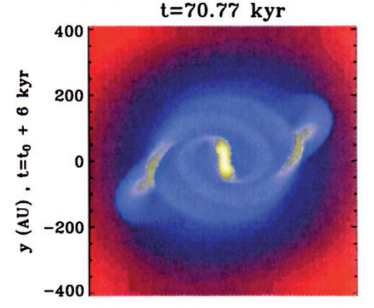

$\mathrm{t}=71.71 \mathrm{kyr}$

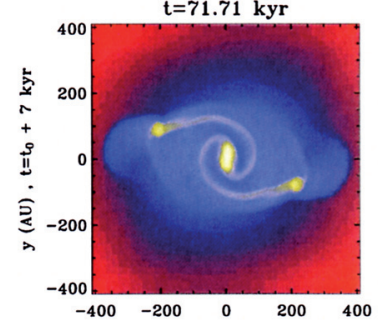

$\mathrm{N}_{\mathrm{P}}=5 \times 10^{5}, \mathrm{~N}_{\mathrm{N}}=50$

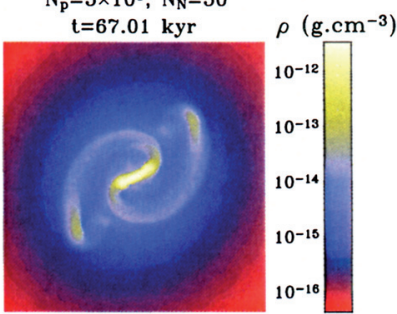

$\mathrm{t}=68.17 \mathrm{kyr}$

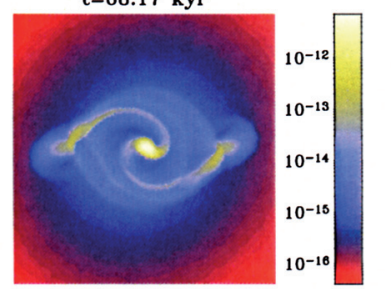

$\mathrm{t}=69.19 \mathrm{kyr}$

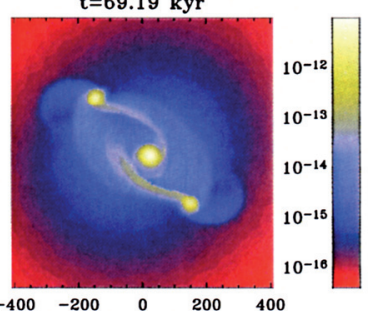

Fig. 8. AMR and SPH calculations density maps in the $x y$-plane at three different times for the case $\alpha=0.50, \beta=0.04$. The times correspond to $t_{0}+5 \mathrm{kyr}, t_{0}+6 \mathrm{kyr}$ and $t_{0}+7 \mathrm{kyr}$, from to bottom, respectively. The AMR calculations plotted on the left column have been run with $\ell_{\min }=7$ and $N_{\mathrm{J}}=15$. The right column shows the results for the SPH calculations with $N_{\mathrm{p}}=5 \times 10^{5}$ and $N_{\mathrm{N}}=50$.

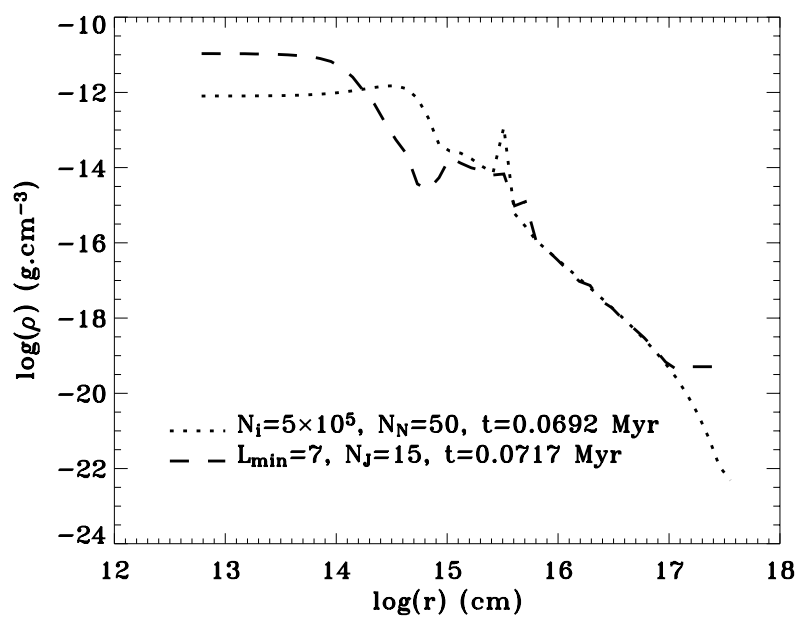

Fig. 9. Density profiles at $t_{0}+7 \mathrm{kyr}$ as a function of the radius, averaged on the equatorial plane, for AMR calculations with $\ell_{\min }=7$ and $N_{\mathrm{J}}=15$ (dashed line), and SPH calculations with $N_{\mathrm{p}}=5 \times 10^{5}$ and $N_{\mathrm{N}}=50$ (dotted line).

SPH calculations with $N_{\mathrm{p}}=5 \times 10^{4}$ diverge quickly compared to two more resolved with $N_{\mathrm{p}}=2 \times 10^{5}$ and $5 \times 10^{5}$. The most resolved AMR and SPH runs show a convergence towards a similar solution. The patterns have the same size and position. The core fragments into a central clump (of size $~ 30 \mathrm{AU}$ ) and two identical outlying clumps (of size $\sim 10 \mathrm{AU}$ ) for AMR calculations. A similar central object is present in the SPH calculations, but the outlying clumps are larger.

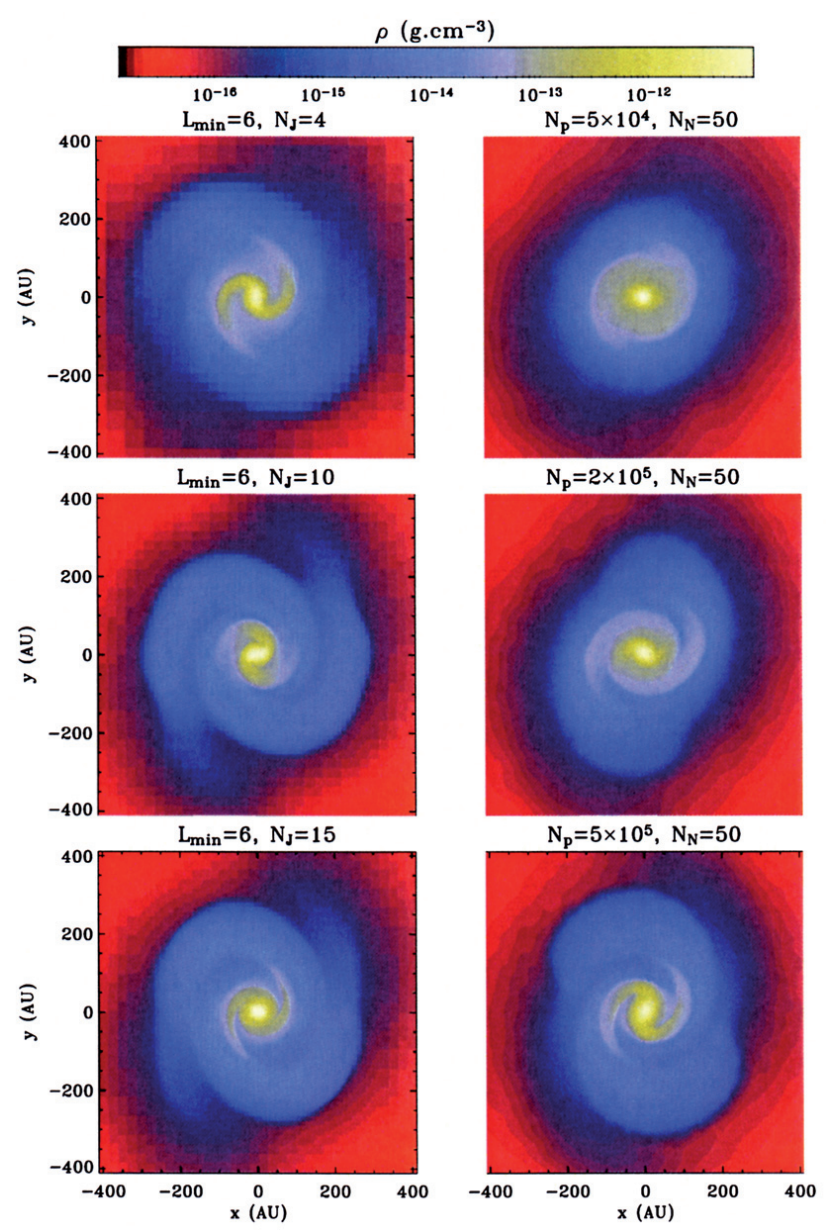

Fig. 10. Density maps in the equatorial plane at $t_{0}+10 \mathrm{kyr}$ for $\alpha=0.65$, $\beta=0.04$ and $A=0.5$. On the left-hand side, we show AMR results with $\ell_{\min }=6$ and, from top to bottom, $N_{\mathrm{J}}=4,10$ and 15 . The right-hand side gives slices of SPH calculations with $N_{\mathrm{N}}=50$ and $N_{\mathrm{p}}=5 \times 10^{4}, 2 \times 10^{5}$ and $5 \times 10^{5}$, from top to bottom.

However, as shown in Appendix C, higher resolution runs show that convergence has not been reached. In that case, one needs either an even better resolution or, alternatively, a more sophisticated numerical scheme.

To conclude our study on core fragmentation, it becomes increasingly difficult to attain convergence in both SPH and AMR simulations as non-linearity increase. Good convergence is found for sufficiently high thermal support. However, for low thermal support (i.e. $\alpha=0.35$ ), convergence is more difficult to achieve and the horizon of predictability becomes short.

\section{Summary and discussion}

We have investigated the effect of changing the numerical resolution in AMR and SPH calculations, on the collapse and the fragmentation of rotating cores.

We show that we reach good convergence between AMR and SPH methods provided one uses sufficient numerical resources. First, we take a simple model to study local angular momentum conservation. The initial study shows that local angular momentum is better conserved with the AMR approach for equivalent computational needs, whereas SPH provides better dynamical times. As shown in Fig. 4a, a smaller number of particles in standard SPH calculations leads to poor local angular momentum conservation. Numerical torques on the rotational axis 

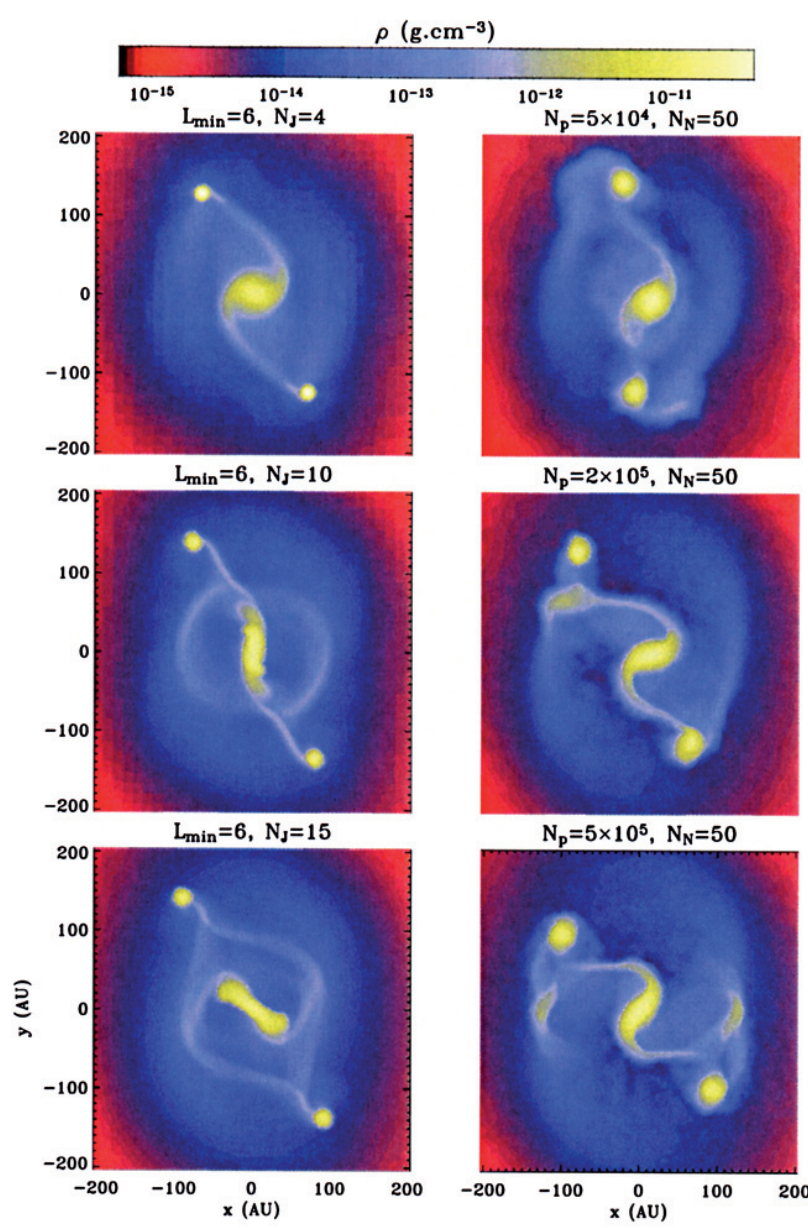

$\mathrm{N}_{\mathrm{P}}=2 \times 10^{5}, \mathrm{~N}_{\mathrm{N}}=50$

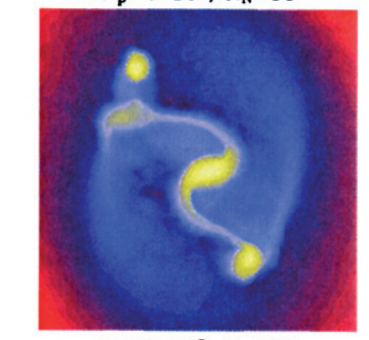

$N_{p}=5 \times 10^{5}, N_{N}=50$

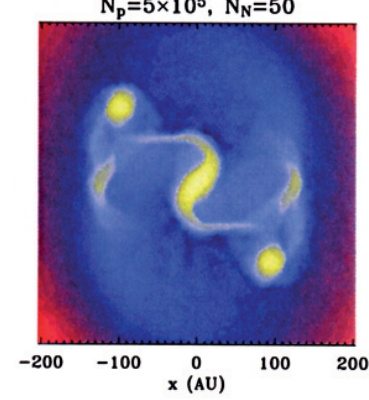

Fig. 11. Density maps in the equatorial plane at $t=t_{0}+3 \mathrm{kyr}$ for the case $\alpha=0.35, \beta=0.04$ and $A=0.1$. On the left side, we give AMR results with $\ell_{\min }=6$ and, from top to bottom, $N_{\mathrm{J}}=4,10$ and 15 . On the right side, we show SPH maps with calculations with $N_{\mathrm{N}}=50$ and, from top to bottom, $N_{\mathrm{p}}=5 \times 10^{4}, N_{\mathrm{p}}=2 \times 10^{5}$ and $N_{\mathrm{p}}=5 \times 10^{5}$.

accumulate onto denser particles, whereas our model remains axisymmetric. In AMR calculations, a poor initial computational domain resolution (i.e. $\ell_{\min }<6$ ) leads to unphysical transfer of gravitational energy to rotational energy (see Fig. 4b). A significant loss of angular momentum will affect fragmentation since less rotational support can balance gravitational collapse. The smallest parameter values set for SPH calculations required to be able to simulate gravitational collapse without significant loss of angular momentum corresponds to a number of $\sim 530$ particles per Jeans mass at the critical density $\rho_{\mathrm{c}}$, i.e. 5 particles per Jeans length. The equivalent minimum resolution criterion for AMR calculations is $\ell_{\min } \gtrsim 6$ and $N_{\mathrm{J}}=4$.

We next investigated fragmentation issues for three different initial condition. For the least prone to fragment case $(\alpha=0.65$, $\beta=0.04$ ), we showed that AMR and SPH methods provide similar results when their respective Jeans resolution criteria are fulfilled. These results agree with semi-analytical criteria on fragmentation derived by Tsuribe \& Inutsuka (1999), for isothermal collapse $(\alpha \lesssim 0.55-0.65 \beta)$. We carefully analyzed simulations completed for the initial conditions parameter values $\alpha=0.5$, $\beta=0.04$. We reached good agreement between AMR calculations with the parameters $\ell_{\text {min }}=6$ and $N_{\mathrm{J}}=15$ and SPH calculations with $N_{\mathrm{p}}=5 \times 10^{5}$ and $N_{\mathrm{N}}=50$, i.e. $\sim 5370$ particles per Jeans mass at critical density $\rho_{\mathrm{c}}$. This parameter set appears to correspond to a lower resolution limit for dense core collapse and fragmentation in SPH and AMR calculations. This is required to reach good agreement in both time and space. Using a lower number of particles or number of points per Jeans length will lead to inaccurate early fragmentation due to numerical effects. Initially, we compared the two converged calculations and, for this specific case, we found good agreement between the two methods (see Fig. 8). For the fragmentation study, the computer time is more expensive for the SPH method due to the lack of use of sink particles. In the case of low thermal support, the dynamic of the flow quickly becomes very non-linear and numerical convergence of the simulation cannot be achieved as easily as for higher thermal support. A statistical analysis over a large number of simulations would be required to assess if convergence can be achieved for fragment masses, sizes and number.

Star formation analyses completed using SPH and AMR simulations provide predictions that, in general, agree. Details are better resolved in AMR calculations thanks to the refinement method based on the local Jeans length, whereas resolution deteriorates with increasing density in standard SPH calculations. Numerical calculations of protostellar collapse should be carefully conducted, with a detailed examination of the effect of numerical resolution. The present work can be used to assess the validity of numerical tools in the study of star formation.

Acknowledgements. Calculations have been performed at the PSMN (ENS Lyon), IDRIS and CCRT (CEA) supercomputating facilities, as well as on the CEMAG computing facility supported by the French ministry of research and education through a Chaire d'Excellence awarded to Steven Balbus. We greatly thank Anthony Whitworth, the referee, for useful comments which have improved the original manuscript. We thank Simon Goodwin for providing the last version of the DRAGON code.

\section{Appendix A: Complementary results on the effect of $N_{\mathrm{N}}$ for SPH calculations for the case: $\alpha=0.5, \beta=0.04$ and $A=0.1$}

Figure A.1 shows SPH calculations run with a constant $N_{\mathrm{p}}=$ $5 \times 10^{5}$ and values of $N_{\mathrm{N}}=30,50,100$ and 200 from top to bottom for two timesteps $\left(t_{0}+5 \mathrm{kyr}\right.$ on the left column and $t_{0}+6 \mathrm{kyr}$ on the right column). These simulations should be compared with simulations presented in Fig. 7. The first relevant result is the fact that increasing $N_{\mathrm{N}}$ speeds up fragmentation. Moreover, there seems to be a similarity between calculations with low $N_{\mathrm{p}} / N_{\mathrm{N}}$ ratio, i.e. $N_{\mathrm{N}}=50, N_{\mathrm{p}}=2 \times 10^{5}$ on one side and $N_{\mathrm{N}}=100$ and $N_{\mathrm{p}}=5 \times 10^{5}$ on the other side. We find the same patterns at different times, postponed when either $N_{\mathrm{p}}$ increases or $N_{\mathrm{N}}$ decreases, the number of resolution elements being equal. This illustrates the compromise between resolution and convergence that must be respected in SPH calculations (Lombardi et al. 1999; Rasio 1999).

\section{Appendix B: Note on the artificial viscosity and numerical diffusion in SPH}

Diffusivity is a well-known drawback of standard SPH. This issue can be reduced using a constant number neighbors, $\Delta N_{\mathrm{N}}=0$ (Attwood et al. 2007), and an advanced scheme for viscosity such as time-dependent viscosity (Morris \& Monaghan 1997). We present here SPH calculations of the collapse of the uniformdensity sphere already studied in Sect. 4, but using another scheme viscosity and/or a constant number of neighbors. This two improvements are straightforward to implement and do not require expensive extra computational costs. Time-dependent 

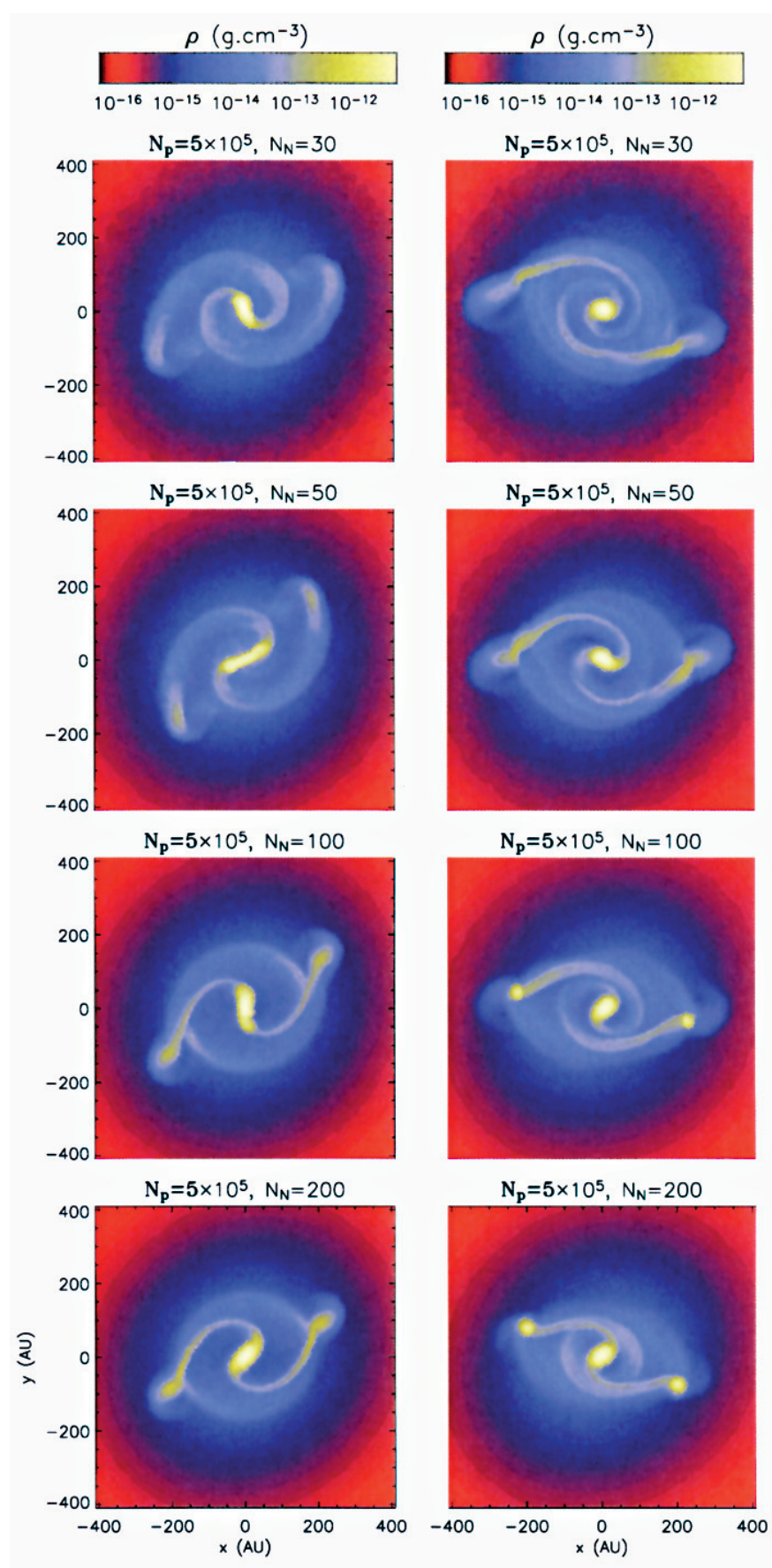

$N_{p}=5 \times 10^{5}, N_{N}=50$

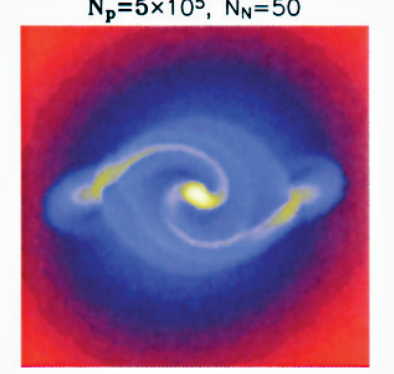

$N_{p}=5 \times 10^{5}, N_{N}=100$

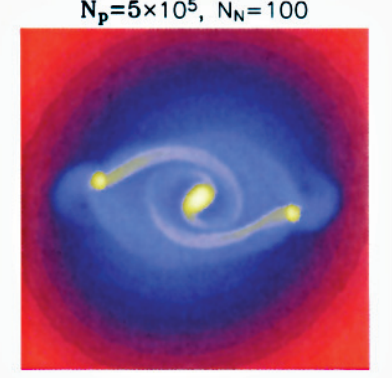

$\mathrm{N}_{\mathrm{p}}=5 \times 10^{5}, \mathrm{~N}_{\mathrm{N}}=200$

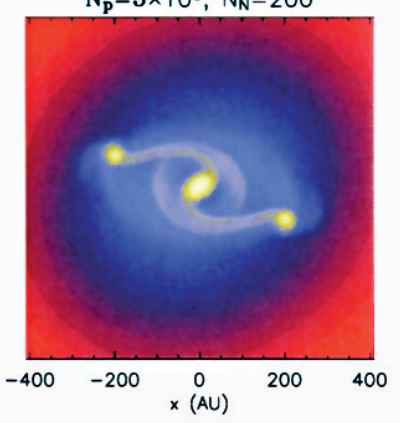

Fig. A.1. Density maps in the equatorial plane at two different times from SPH calculations with $\alpha=0.50, \beta=0.04$ and $N_{\mathrm{p}}=5 \times 10^{5}$. The left column shows density maps for calculations with $N_{\mathrm{N}}=30,50,100$ and 200 from top to bottom, respectively, at $t=t_{0}+5 \mathrm{kyr}$. The right column represents the same calculations at $t=t_{0}+6 \mathrm{kyr}$.

viscosity calculations have been completed for $\alpha^{\star}=0.1$, and an e-folding constant equal to 0.15 .

Figure B.1a shows the averaged ratio between angular momentum $J(t)$ at time $t_{0}$, and initial angular momentum $J_{0}$ as a function of particles (ordered in decreasing density) for SPH calculations run with $N_{\mathrm{p}}=5 \times 10^{5}$ and $N_{\mathrm{N}}=50$. In addition, the improvement on viscosity and number of neighbors above mentioned are either turned on or not. It is clear that time-dependent viscosity can better conserve angular momentum for the denser particles. Hence, less angular momentum is transported to the outer part of the core. This is confirmed in Fig. B.1b where we
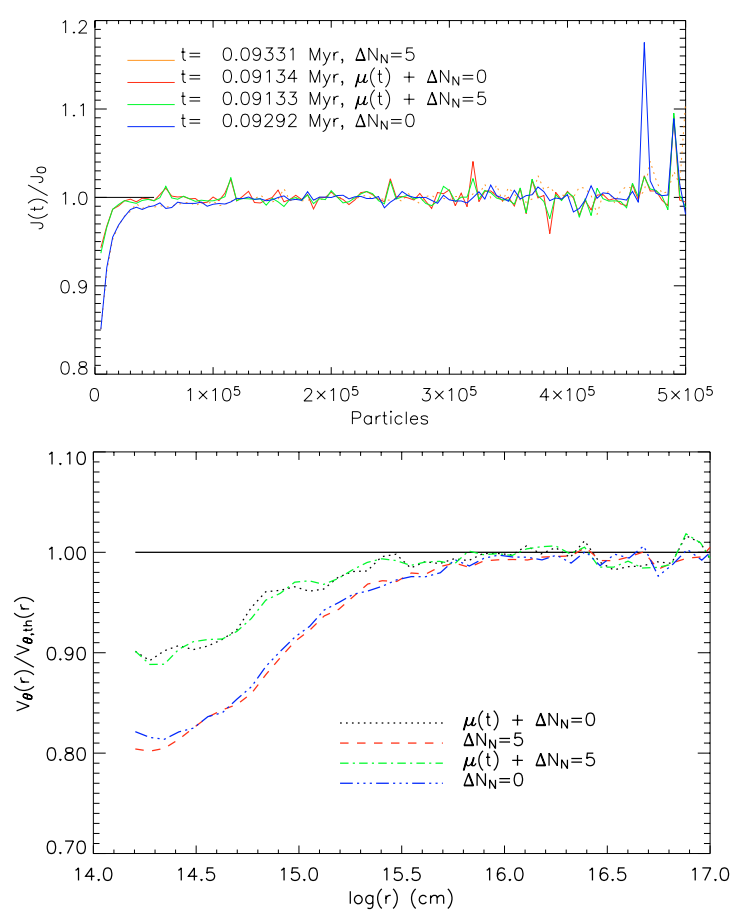

Fig. B.1. Top plot a) same as Fig. 2 with different $\Delta N_{\mathrm{N}}$ and artificial viscosity scheme. $\mu(t)$ indicates the use of time-dependent viscosity instead of the standard artificial viscosity scheme in the paper. Bottom plot b) same as Fig. 5 for the above mentioned SPH calculations.

plot the ratio between angular momentum at time $t_{0}$ and initial angular momentum, as a function of the radius, for the same calculations. Local angular momentum conservation is increased by $10 \%$ in the inner part. However, keeping the number of neighbors constant does not improve local angular momentum conservation because the system evolves only over about one freefall time, whereas Attwood et al. (2007) shows that dissipation becomes significant after a few free-fall times. All of these improvements of standard SPH will strengthen convergence with the AMR.The particle-splitting appears to be the most promising one.

\section{Appendix C: Note on the diffusion of the numerical schemes in AMR}

A key ingredient of the AMR method is the numerical scheme used to compute flux at the grid's interfaces. In this paper, we use a Lax-Friedrich (hereafter LF) Riemann solver designed for MHD calculations (Fromang et al. 2006). However, the LF scheme is known to be a diffusive scheme. In this appendix, we present AMR calculations carried out with, on one hand, the LF scheme and, on the other hand, a Roe scheme. The Roe scheme is less diffusive than LF, and this could dramatically affect the fragmentation issue.

\section{C.1. Case $\alpha=0.5, \beta=0.04$}

Figure C.1 shows density maps on the equatorial at three different timesteps for two AMR calculations run with the same numerical parameters, i.e. $\ell_{\min }=6$ and $N_{\mathrm{J}}=15$, but with a different solver, i.e. the LF one on the left column and the Roe one on the right column. Results are quite similar, AMR calculations are in good agreement for this critical case with the two solvers. Since less angular momentum has been locally lost or transported with 

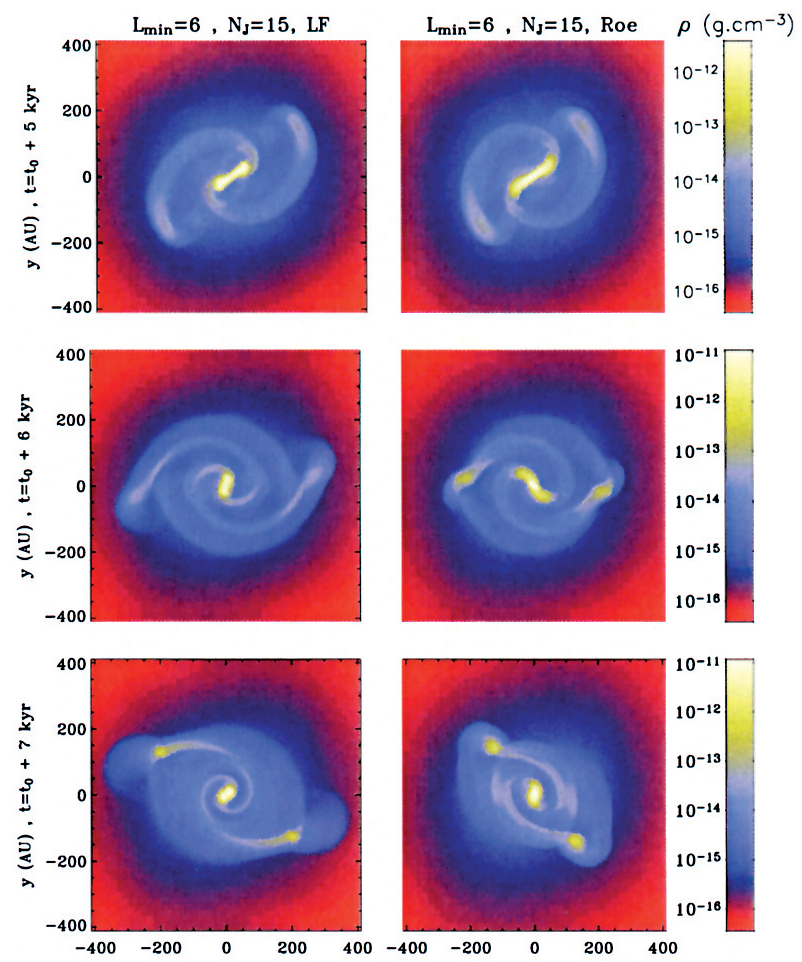

Fig. C.1. Density maps in the equatorial plane for the case $\alpha=0.5$, $\beta=0.04$ at, from top to bottom, $t=t_{0}+5 \mathrm{kyr}, t=t_{0}+6 \mathrm{kyr}$ and $t=$ $t_{0}+7 \mathrm{kyr}$. On the left-hand side, AMR results with Lax-Friedrich solver and $\ell_{\min }=6, N_{\mathrm{J}}=15$ are reported and AMR results of calculations with the same parameters but with a Roe solver are given on the right column.

the Roe scheme, the core is smaller and the fragments are closer to the central object. This brings support to the fact that we find good convergence between AMR and SPH calculations for this case.

\section{C.2. Case $\alpha=0.35, \beta=0.04$}

Figure C.2 shows density maps on the equatorial plane at $t_{0}+$ $2 \mathrm{kyr}$ (right column) and $t_{0}+3 \mathrm{kyr}$ for three simulations of the case $\alpha=0.35, \beta=0.04$ with numerical parameters $\ell_{\min }=6$ and $N_{\mathrm{J}}=12$ (top and bottom maps, LF and Roe schemes) and $\ell_{\text {min }}=7$ and $N_{\mathrm{J}}=15$ (middle row, LF scheme). We remark that in Fig. 11 , the case $\ell_{\min }=6$ and $N_{\mathrm{J}}=15$ with the LF solver has been displayed. The two calculations with the same numerical parameters differ, according to the numerical scheme used. The fragmentation process changes: one achieves a configuration central object + two satellites with the LF scheme whereas we get a binary system resulting from the fragmentation of the central object with the Roe scheme. If we improve the initial sphere resolution in LF calculations (i.e. $\ell_{\min }=7, N_{\mathrm{J}}=15$ ), we converge to the results obtained with the Roe scheme, i.e. a central binary system, with a value $\ell_{\min }<7$. We know that angular momentum is well conserved using the Roe scheme or the LF scheme with $\ell_{\text {min }}=7$, so it seems that calculations lead to a different core fragmentation because of their less accurate angular momentum conservation. Since we use a small thermal support, it is easy to reach another fragmentation configuration, these processes being highly non-linear.
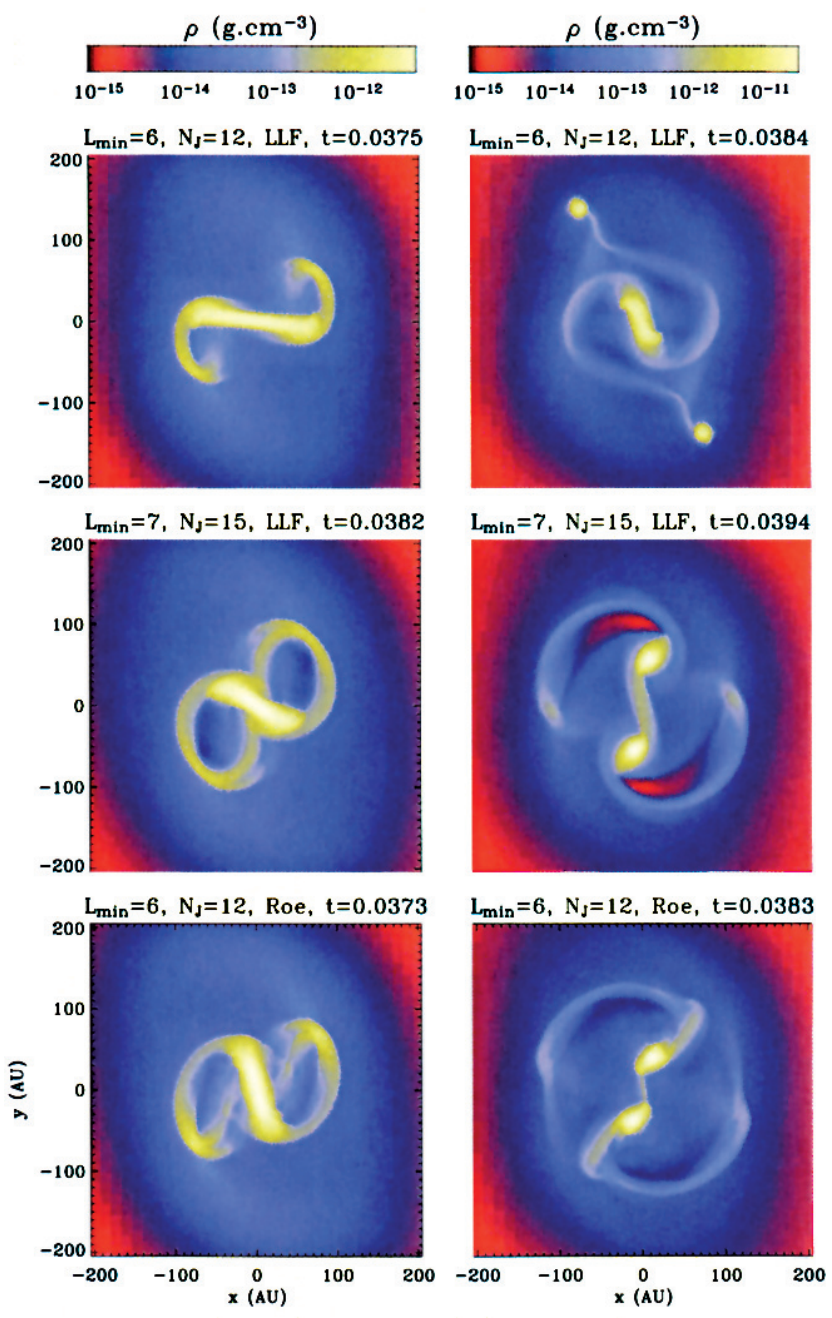

Fig. C.2. Density maps in the equatorial plane for the case $\alpha=0.35$, $\beta=0.04$ at $t=t_{0}+2 \mathrm{kyr}$ on the left-hand side and $t=t_{0}+3 \mathrm{kyr}$ on the right-hand side. For the two upper rows, we plot results for AMR calculations with $\left(\ell_{\min }=6, N_{\mathrm{J}}=12\right),\left(\ell_{\min }=7, N_{\mathrm{J}}=15\right)$ and our usual Lax-Friedrich scheme. The bottom row gives the results for calculations conducted with $\left(\ell_{\min }=6, N_{\mathrm{J}}=12\right)$ too, but with a Roe solver. Times are given in Myr.

\section{Appendix D: Note on SPH sink particles}

The introduction of sink particles is a widely used method to obtain a compromise between good resolution and acceptable timestep in SPH methods. Creating a sink particle enables the Courant condition on the particle timesteps to be relaxed.

The density level $\rho_{\text {sink }}$ at which a sink particle is created has to be chosen with care. In the previous SPH calculations, no sink particles were used. We focus on the highly non-linear fragmentation case, $\alpha=0.35, \beta=0.04$ and $A=0.1$ to present $\mathrm{SPH}$ calculations carried out with various sink densities.

Figure D.1 shows calculations carried out with three different densities for the creation of sink particles, $\rho_{\text {sink }}$, namely $1 \times 10^{-10} \mathrm{~g} \mathrm{~cm}^{-3}$ (resulting in no sink creation), $1 \times 10^{-11} \mathrm{~g} \mathrm{~cm}^{-3}$ and $3 \times 10^{-12} \mathrm{~g} \mathrm{~cm}^{-12}$, from top to bottom, and the same number of SPH particles and neighbors, $N_{\mathrm{p}}=5 \times 10^{5}$ and $N_{\mathrm{N}}=50$. The left-hand side reports results at $t_{0}+1 \mathrm{kyr}$ and the righthand column results at $t_{0}+3 \mathrm{kyr}$. The CPU time is about $28 \%$ smaller for the case $\rho_{\text {sink }}=3 \times 10^{-12} \mathrm{~g} \mathrm{~cm}^{-3}$ than for the case $\rho_{\text {sink }}=1 \times 10^{-10} \mathrm{~g} \mathrm{~cm}^{-3}$, but the dense core resulting at $t_{0}+3 \mathrm{kyr}$ is different. The upper row corresponds to 

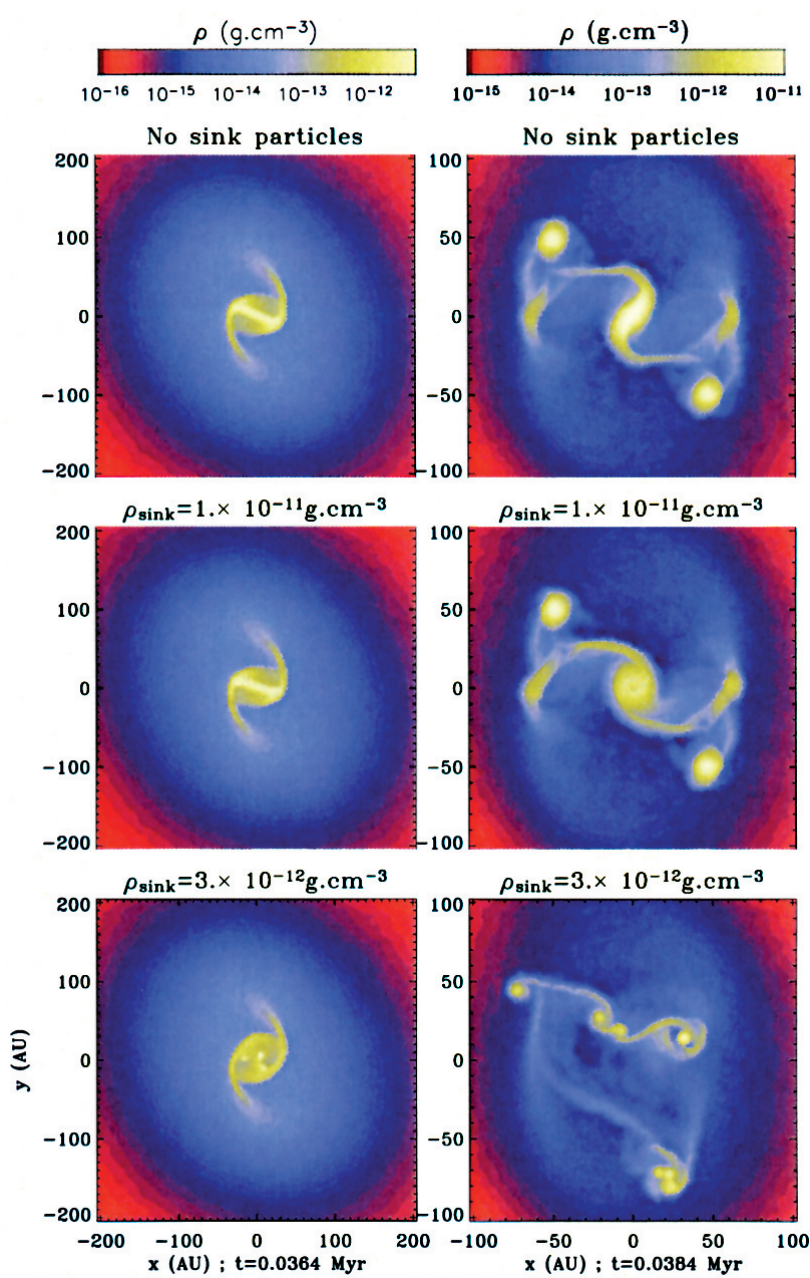

Fig. D.1. Density maps in the equatorial plane for the case $\alpha=0.35$, $\beta=0.04$ at $t=t_{0}+1 \mathrm{kyr}$ on the left-hand side and $t=t_{0}+3 \mathrm{kyr}$ on the right-hand side. 9 sink particles have been created on the bottom figures, affecting the whole dynamic of the dense core.

calculations without creation of sink particles, whereas one sink particle has been created with $\rho_{\text {sink }}=1 \times 10^{-11} \mathrm{~g} \mathrm{~cm}^{-3}$ and 9 with $\rho_{\text {sink }}=3 \times 10^{-12} \mathrm{~g} \mathrm{~cm}^{-3}$. It is easy to see that, even if only one sink particle is created, the complete dynamic of the flow is affected particularly in the central region.
In conclusion, it is clear that sink particles should be handled with great care. A fair comparison should also compare these SPH calculations with AMR ones including sink particles. Such studies are in progress.

\section{References}

Arreaga-García, G., Klapp, J., Sigalotti, L. D. G., \& Gabbasov, R. 2007, ApJ, 666,290

Attwood, R. E., Goodwin, S. P., \& Whitworth, A. P. 2007, A\&A, 464, 447

Bate, M. R., \& Burkert, A. 1997, MNRAS, 288, 1060

Bate, M. R., Bonnell, I. A., \& Price, N. M. 1995, MNRAS, 277, 362

Berger, M. J., \& Oliger, J. 1984, J. Chem. Phys, 53, 484

Bonnell, I. A. 1994, MNRAS, 269, 837

Boss, A. P., \& Bodenheimer, P. 1979, ApJ, 234, 289

Dehnen, W. 2001, MNRAS, 324, 273

Frenk, C. S., White, S. D. M., Bode, P., et al. 1999, ApJ, 525, 554

Fromang, S., Hennebelle, P., \& Teyssier, R. 2006, A\&A, 457, 371

Gingold, R. A., \& Monaghan, J. J. 1977, MNRAS, 181, 375

Gingold, R. A., \& Monaghan, J. J. 1983, MNRAS, 204, 715

Goodwin, S. P., Whitworth, A. P., \& Ward-Thompson, D. 2004, A\&A, 414, 633

Hennebelle, P., Whitworth, A. P., Cha, S.-H., \& Goodwin, S. P. 2004, MNRAS, 348,687

Hernquist, L. 1987, ApJS, 64, 715

Hosking, J. G., \& Whitworth, A. P. 2004, MNRAS, 347, 1001

Hubber, D. A., Goodwin, S. P., \& Whitworth, A. P. 2006, A\&A, 450, 881

Inutsuka, S. 1994, Mem. Soc. Astron. Ital., 65, 1027

Khokolov, A. M. 1998, J. Chem. Phys., 143, 519

Kitsionas, S., \& Whitworth, A. P. 2002, MNRAS, 330, 129

Klein, R. I., Inutsuka, S.-I., Padoan, P., \& Tomisaka, K. 2007, in Protostars and Planets V, ed. B. Reipurth, D. Jewitt, \& K. Keil, 99

Larson, R. B. 1969, MNRAS, 145, 271

Lombardi, J. C., Sills, A., Rasio, F. A., \& Shapiro, S. L. 1999, Journal of Computational Physics, 152, 687

Lucy, L. B. 1977, AJ, 82, 1013

Masunaga, H., \& Inutsuka, S.-i. 2000, ApJ, 531, 350

Matsumoto, T., \& Hanawa, T. 2003, ApJ, 595, 913

Morris, J. P., \& Monaghan, J. J. 1997, J. Comput. Phys., 136, 41

Nelson, A. F. 2006, MNRAS, 373, 1039

Penston, M. V. 1969, MNRAS, 144, 425

Rasio, F. A. 1999, Particle Methods in Astrophysical Fluid Dynamics

Shu, F. H. 1977, ApJ, 214, 488

Teyssier, R. 2002, A\&A, 385, 337

Tohline, J. E. 1982, Fundamentals of Cosmic Physics, 8, 1

Truelove, J. K., Klein, R. I., McKee, C. F., et al. 1997, ApJ, 489, L179

Truelove, J. K., Klein, R. I., McKee, C. F., et al. 1998, ApJ, 495, 821

Tsuribe, T., \& Inutsuka, S.-I. 1999, ApJ, 526, 307

Turner, J. A., Chapman, S. J., Bhattal, A. S., et al. 1995, MNRAS, 277, 705

Whitworth, A. P., \& Clarke, C. J. 1997, MNRAS, 291, 578

Whitworth, A. P., Bhattal, A. S., Turner, J. A., \& Watkins, S. J. 1995, A\&A, 301, 929

Ziegler, U. 2005, A\&A, 435, 385 